\title{
An operational construction of the sum of two non-commuting observables in quantum theory and related constructions
}

\author{
Nicolò Drago ${ }^{1} \cdot$ Sonia Mazzucchi ${ }^{1} \cdot$ Valter Moretti $^{1}$
}

Received: 21 April 2020 / Revised: 25 August 2020 / Accepted: 18 September 2020 /

Published online: 4 October 2020

(c) The Author(s) 2020

\begin{abstract}
The existence of a real linear space structure on the set of observables of a quantum system-i.e., the requirement that the linear combination of two generally noncommuting observables $A, B$ is an observable as well-is a fundamental postulate of the quantum theory yet before introducing any structure of algebra. However, it is by no means clear how to choose the measuring instrument of a general observable of the form $a A+b B(a, b \in \mathbb{R})$ if such measuring instruments are given for the addends observables $A$ and $B$ when they are incompatible observables. A mathematical version of this dilemma is how to construct the spectral measure of $f(a A+b B)$ out of the spectral measures of $A$ and $B$. We present such a construction with a formula which is valid for general unbounded self-adjoint operators $A$ and $B$, whose spectral measures may not commute, and a wide class of functions $f: \mathbb{R} \rightarrow \mathbb{C}$. In the bounded case, we prove that the Jordan product of $A$ and $B$ (and suitably symmetrized polynomials of $A$ and $B$ ) can be constructed with the same procedure out of the spectral measures of $A$ and $B$. The formula turns out to have an interesting operational interpretation and, in particular cases, a nice interplay with the theory of Feynman path integration and the Feynman-Kac formula.
\end{abstract}

Keywords Non-commuting observables · Unbounded self-adjoint operators · Spectral measures $\cdot$ Foundations of quantum theories

Mathematics Subject Classification 46L60 - 46L65 - 47L99 · 46N50 · 81T05

Valter Moretti

valter.moretti@unitn.it

Nicolò Drago

nicolo.drago@unitn.it

Sonia Mazzucchi

sonia.mazzucchi@unitn.it

1 Department of Mathematics, University of Trento, and INFN-TIFPA, Via Sommarive 14, Povo, 38123 Trento, Italy 


\section{Contents}

1 Introduction . . . . . . . . . . . . . . . . . . . . . . . . . . . . . 3198

2 From $\mathbb{C}^{n}$ to an infinite-dimensional Hilbert space . . . . . . . . . . . . . . . . . . . 3202

2.1 The finite-dimensional Hilbert space case ． . . . . . . . . . . . . . . . . . . . . 3202

2.2 The unital Banach algebra of complex Borel measures . . . . . . . . . . . . . . . 3205

2.3 Regularized products of non-commuting PVMs of self-adjoint operators . . . . . . . . 3206

2.4 Main result . . . . . . . . . . . . . . . . . . . . . . . . . . . . . . 3210

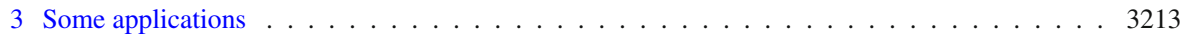

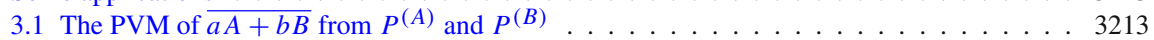

3.2 Relation with Feynman integration . . . . . . . . . . . . . . . . . . . 3216

3.3 (Semi)bounded A, B, Jordan product, polynomials, Feynman-Kac formula . . . . . . 3218

4 Counterexamples . . . . . . . . . . . . . . . . . . . . . . . 3223

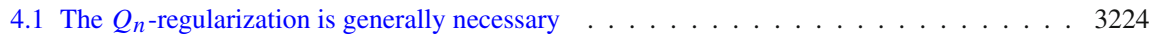

4.2 The sequence of measures $v_{\phi, \psi}^{(N)}$ generally does not admit a (projective) limit . . . . . . 3225

4.3 Convergence of $\left\{\mu_{\phi, \psi}^{(N, n)}\right\}_{n \in \mathbb{N}}$ in $\mathscr{M}\left(\mathbb{R}^{2 N}\right)$ for commuting PVMs: a counterexample . . . 3227

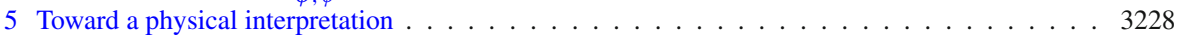

5.1 Non-commutativity and quantum interference terms . . . . . . . . . . . . . . 3228

5.2 Sum over all possible histories . . . . . . . . . . . . . . . . . 3233

6 Summary . . . . . . . . . . . . . . . . . . . . . . . . . . 3234

Appendix A: Proof of some propositions . . . . . . . . . . . . . . . . . 3235

Appendix B: Spectral representation theorem . . . . . . . . . . . . . . . . . . . . 3237

Appendix C: Projective limit of complex measures . . . . . . . . . . . . . . . . . . . . 3239

References. . . . . . . . . . . . . . . . . . . . 3240

\section{Introduction}

The elementary formulation of Quantum Theory in a complex separable Hilbert space $\mathrm{H}$ can be described as a non-Boolean probability theory. There, quantum states $\mu$ are probability measures over the orthomodular separable $\sigma$-complete lattice $\mathscr{L}(\mathrm{H})$ of orthogonal projectors (the order relation being the standard inclusion of projection subspaces) which generalizes the notion of $\sigma$-algebra (see, e.g., $[4,18,22,25,27,42]$ ). The Hilbert space $\mathrm{H}$ depends on the physical system $S$ one intends to study and the elements of $\mathscr{L}(\mathrm{H})$ physically represent elementary propositions of $S$, also known as YES-NO elementary observables or tests about $S$. They are therefore assumed to have a definite operational meaning in terms of experimental devices. Commutativity of $P, Q \in \mathscr{L}(\mathrm{H})$ has the physical meaning of simultaneous measurability of the elementary observables represented by $P$ and $Q$. In that case, $P$ and $Q$ are said to be compatible.

The issue we want to address in this work concerns the set $\mathcal{O}_{S}$ of observables of $S$. An observable $A$ is primarily a collection of elementary propositions $P_{E}^{(A)}$ labeled by real Borel sets $E \in \mathscr{B}(\mathbb{R})$. The operator $P_{E}^{(A)}$ admits an explicit operational interpretation in terms of the statement "the outcome of the measurement of the observable $A$ belongs to $E$." We stress that there must be one (or several) measuring instrument(s) associated to the class $\left\{P_{E}^{(A)}\right\}_{E \in \mathscr{B}(\mathbb{R})}$. Sound physical and logical arguments (see, e.g., $[18,25,42]$ ) require that the collection $\left\{P_{E}^{(A)}\right\}_{E \in \mathscr{B}(\mathbb{R})}$ defines a $\sigma$-Boolean algebra homomorphism form $\mathscr{B}(\mathbb{R})$ to $\mathscr{L}(\mathrm{H})$. In terms more familiar to physicists, $\left\{P_{E}^{(A)}\right\}_{E \in \mathscr{B}(\mathbb{R})}$ is a projector-valued measure (PVM) also known as spectral measure. 
Among them, compatible observables $A, B$ are by definition those whose associated PVMs are made of pairwise compatible projectors, i.e., $P_{E}^{(A)} P_{F}^{(B)}=P_{F}^{(B)} P_{E}^{(A)}$ for all $E, F \in \mathscr{B}(\mathbb{R})$.

At this juncture, the spectral machinery establishes that there is a one-to-one correspondence between PVMs over $\mathrm{H}$ and (generally unbounded) self-adjoint operators $A: D(A) \rightarrow \mathrm{H}$. As a matter of fact, the following holds

$$
A=\int_{\mathbb{R}} \lambda \mathrm{d} P^{(A)}(\lambda) .
$$

We conclude that $\mathcal{O}_{S}$ is made of some self-adjoint operators $A: D(A) \rightarrow \mathrm{H}$ and the natural question emerges about how large $\mathcal{O}_{S}$ is in the whole set of self-adjoint operators over $\mathrm{H}$.

As a matter of fact, it is generally assumed as a further postulate that $\mathcal{O}_{S}^{(b)}:=$ $\mathcal{O}_{S} \cap \mathfrak{B}(\mathrm{H})$ is a linear subspace ${ }^{1}$ of the real linear space of bounded ${ }^{2}$ self-adjoint operators of $\mathfrak{B}(\mathrm{H})$. This structure is later enriched $[12,18,25,40])$ by adding in particular the so-called (non-associative) Jordan product, and assuming suitable (weak) topological features compatible with the spectral machinery. The final construction turns out to be a concrete Jordan $W$-algebra in $\mathfrak{B}(\mathrm{H})$ or, more generally, a concrete von Neumann algebra in $\mathfrak{B}(\mathrm{H})$ when complex combination of observables is permitted. These structures are actually difficult to physically justify a priori. If assumed, they, however, promote the theory to a very high level of effectiveness in physics $a$ posteriori.

Already sticking to the linear structure of the set of bounded observables, we argue that a long-standing (see, e.g., [9,39]) open issue pops out, with a twofold nature, both physical and mathematical. Suppose that $A, B \in \mathcal{O}_{S}^{(b)}$ and that we know their respective measuring instruments. If we pick out $a, b \in \mathbb{R}$, in spite of the postulate about the linear structure of $\mathcal{O}_{S}^{(b)}$, there is no general way to associate a measuring instrument to $a A+b B$ out of those of $A$ and $B$. This obstruction is valid unless the observables $a A$ and $b B$ are compatible ${ }^{3}$. In that case, the instruments corresponding to the joint $P V M$ of $P^{(a A)}$ and $P^{(b B)}$ can be exploited.

The mathematical version of the issue raised above is that no general formula is known determining the operator $f(a A+b B)$-and every projector $P_{E}^{(a A+b B)}$ in particular - as a function of $P^{(A)}$ and $P^{(B)}$ when these PVMs do not commute. This work addresses the outlined problem from the mathematical side. We will prove-c.f.

\footnotetext{
${ }^{1}$ It is finally assumed that in the absence of superselection rules and gauge symmetries, the complex span of the elements in $\mathcal{O}_{S}^{(b)}$ amounts to the whole $\mathfrak{B}(\mathrm{H})$; otherwise, it is a von Neumann algebra in $\mathrm{H}$.

2 Notice that $\mathcal{O}_{S}^{(b)}$ suffices to recover all physical information carried by $\mathcal{O}_{S}$. Indeed, for all $A \in \mathcal{O}_{S}$ one has $A_{N}:=\int_{[-N, N]} \lambda \mathrm{d} P^{(A)}(\lambda) \in \mathfrak{B}(\mathrm{H})(N \in \mathbb{N})$ as well as $\left(A_{N}-A\right) \psi \rightarrow 0$ as $N \rightarrow+\infty$ for all $\psi \in D(A)$. The operator $A_{N}$ can be interpreted as the same observable $A$ measured with instruments whose range is restricted to $[-N, N]$.

3 The problem tackled in this work is a consequence of the existence of incompatible observables so that it does not arise in classical physics. Given a classical system $S$, for instance, described in its phase space, there is a minimal set of observables such that all remaining observables are functions of them. Therefore, measuring the first ones exactly amounts to measuring all observables of $S$.
} 
(32) and Theorem 2.5-that for a suitable class of continuous functions $f: \mathbb{R} \rightarrow \mathbb{R}$, the following formula holds true:

$$
f(\overline{a A+b B})=s-\lim _{N \rightarrow+\infty} \int_{\mathbb{R}^{2 N}} f\left(\frac{1}{N} \sum_{n=1}^{N}\left(a \lambda_{n}+b \mu_{n}\right)\right) \mathrm{d} P_{\lambda_{1}}^{(A)} \mathrm{d} P_{\mu_{1}}^{(B)} \cdots \mathrm{d} P_{\lambda_{N}}^{(A)} \mathrm{d} P_{\mu_{N}}^{(B)}
$$

This way the self-adjoint operator $f(a A+b B)$ can be computed out of the PVMs of the self-adjoint operators $A$ and $B$. Equation (2) is valid for generally non-commuting $P^{(A)}$ and $P^{(B)}$, also in the case of unbounded self-adjoint operators $A$ and $B$ under the condition that $a A+b B$ is essentially self-adjoint over $D(A) \cap D(B)$. The class of functions $f$ is sufficiently large to include all polynomials when $A$ and $B$ are bounded, in particular $a A+b B$ itself.

As one of the various by-products of the above-mentioned result for the case of $A$ and $B$ bounded, we also find an identity connecting the Jordan product $A \circ B:=$ $\frac{1}{2}(A B+B A)$ to the generally non-commuting PVMs $P^{(A)}$ and $P^{(B)}-c f$. Theorem 3.6.

Comparison with existing literature The assumption of the existence of a real linear space structure over the set of observables is crucial also in much more abstract, and apparently more operational, formulations of the quantum theory from [22] to [40]. Therein, the primary object is an abstract Jordan Banach ${ }^{*}$-algebra (or directly a $C^{*}$ algebra), out of which the Hilbert space formulation is recovered a posteriori through the GNS construction. Observables are defined in terms of their expectation values (here properly interpreted as states), and it is postulated that given a pair of observables $A$ and $B$ and a corresponding pairs of reals $a, b$, there is an observable denoted by $a A+b B$ whose expectation values are the linear combination of the expectation values of $A$ and $B$ with coefficients $a$ and $b$. Though this observable is proved to be unique (because the states are reasonably supposed to separate the observables), no ideas are supplied to solve the problem we pointed out: how we can measure $a A+b B$ if we know how to measure $A$ and $B$ when they are incompatible.

From the mathematical side, our results are entangled with the construction of a functional calculus for non-commuting operators. Several rigorous results exist in the literature about this subject. However, to the best of our knowledge, almost all are concentrated on two physically well-motivated approaches: the generalization of Feynman's functional calculus for T-ordered products of operators and extensions of Weyl calculus - see $[13,15,16,28]$ for exhaustive reviews on these approaches-with several original contributions from outstanding authors $[3,23,26]$. The mathematical technology, the types of functional spaces and the interactions with some other mathematical objects like the Feynman-Kac integral share some similarities with the content of in this work-c.f. Sect. 3.2. However, to authors' knowledge, none of those papers presents an integral expansion like our formula (2) where (a) a joint integral with respect to the non-commuting spectral measures of the involved operators shows up and (b) the physically suggestive averaged value of spectral parameters takes placec.f. Sect. 5 .

Structure of the work The paper is organized as follows. After recalling some general facts and the general notation used throughout, in Sect. 2 we tackle the problem raised 
above first in the finite-dimensional case and next in the infinite-dimensional case. Dealing with a pair of self-adjoint operators $A, B$ in a complex Hilbert space and a continuous bounded function $f: \mathbb{R} \rightarrow \mathbb{C}$ of a certain class, our main result consists of a formula where the operator $f(a A+b B)$ is written as the (strong) limit of a sequence of certain operator-valued integrals whose measure is an alternating product of spectral measures of $A$ and $B$. As a matter of fact, we prove Eq. (9) in the finitedimensional case and Eq. (32) in the much harder infinite-dimensional case. Section 3 is devoted to study some applications: In particular, we present a (fairly explicit) construction for the PVM of $a A+b B$ and the Jordan product $A \circ B$ in terms of the PVM of $A$ and $B$. Eventually, we discuss the nice interplay with both the theory of Feynman integration in the phase space and the so-called Feynman-Kac formula. Section 4 contains some counterexamples which demonstrate how part of the structure introduced in the infinite-dimensional case cannot be improved. In Sect. 5, we discuss the physical interpretation of the constructed formalism in the finite-dimensional case where no technical issues take place, allowing us to focus directly on physics. A short summary ends the paper.

General conventions and notation We adopt throughout the paper the standard definition of complex measure [35], as a map $\mu: \Sigma \rightarrow \mathbb{C}$ which is unconditionally $\sigma$ additive over the $\sigma$-algebra $\Sigma$. With this definition, the total variation $\|\mu\|:=|\mu|(\Sigma)$ turns out to be finite. By $\mathscr{B}(X)$, we denote the Borel $\sigma$-algebra over a topological space $X$ and $L^{p}(X, v)$ denotes the standard $L^{p}$ space with respect to a positive Borel measure $v$ [35] (in case $v$ is complex, the associated $L^{p}$ spaces are those referred to $|v|$ ). By $L^{p}(\mathbb{R}, \mathrm{d} x)$ (also with $\mathrm{d} y$ in place of $\mathrm{d} x$ ), we mean the $L^{p}$ space with respect to Lebesgue measure (viewed as a Borel measure) on $\mathbb{R}$. In case the measure $v$ is defined on a generic $\sigma$-algebra $\Sigma$ over the set $X$, we use the more precise notation $L^{p}(X, \Sigma, v)$. If $E \subset X$, for any given set $X$, then the indicator function $1_{E}$ of $E$ is defined as $1_{E}(x):=1$ if $x \in E$ and $1_{E}(x)=0$ if $x \in X \backslash E$.

A Hilbert space is always assumed to be complex. We assume the usual convention concerning standard domains of composition of operators over a Hilbert space $\mathrm{H}$, $A: D(A) \rightarrow \mathrm{H}$ and $B: D(B) \rightarrow \mathrm{H}$ with $D(A), D(B) \subset \mathrm{H}$ linear subspaces: (i) $D(B A):=\{\psi \in D(A) \mid A \psi \in D(B)\}$; (ii) $D(A+B):=D(A) \cap D(B)$; (iii) $D(a A):=$ $D(A)$ for $a \in \mathbb{C} \backslash\{0\}$ and $D(0 A):=\mathrm{H}$. These requirements yield in particular $D(a A+$ $b B)=D(A) \cap D(B)$ if $a b \neq 0$. We define $A^{k}:=A \cdots(k$ times $) \cdots A$ if $k=1,2, \ldots$ and $A^{0}:=I$. By $A \subset B$ we mean that $D(A) \subset D(B)$ and $\left.B\right|_{D(A)}=A$.

We denote by $A^{*}: D\left(A^{*}\right) \rightarrow \mathrm{H}$ the adjoint operator of a densely defined operator $A: D(A) \rightarrow \mathrm{H}$ and by $\bar{A}$ its closure. A densely defined operator $A$ is self-adjoint if $A^{*}=A$. The operator $A$ is essentially self-adjoint if $A \subset A^{*}$ and $\bar{A}$ is self-adjoint. We denote by $\mathfrak{B}(\mathrm{H})$ the unital $C^{*}$-algebra of bounded everywhere defined operators over the Hilbert space $\mathrm{H}$, and $\mathscr{L}(\mathrm{H}) \subset \mathfrak{B}(\mathrm{H})$ denotes the lattice of orthogonal projectors: $P=P^{*}=P P$.

The spectrum of an operator $A$ is always denoted by $\sigma(A)$. According to the spectral theory (details, e.g., in [25,34]), if $T: D(T) \rightarrow \mathrm{H}$ is a generally unbounded selfadjoint operator in $\mathrm{H}$ and $g: \mathbb{R} \rightarrow \mathbb{C}$ is measurable, then one has 


$$
g(T):=\int_{\mathbb{R}} g(\tau) \mathrm{d} P^{(T)}(\tau), \quad D(g(T)):=\left\{\left.\psi \in \mathrm{H}\left|\int_{\mathbb{R}}\right| g(\tau)\right|^{2} \mathrm{~d} \mu_{\psi, \psi}^{(T)}(\tau)<+\infty\right\} .
$$

Above, $\mu_{\phi, \psi}^{(T)}(E):=\left\langle\phi \mid P_{E}^{(T)} \psi\right\rangle$ if $E \in \mathscr{B}(\mathbb{R})$ defines a regular complex Borel measure, which is positive if $\psi=\phi$, and $\left\{P_{E}^{(T)}\right\}_{E \in \mathscr{B}(\mathbb{R})}$ is the projection-valued measure (PVM) - also known as a spectral measure-uniquely associated with $T$. The right-hand side of (3) can be defined [36] ${ }^{4}$ as the unique operator $T$ on $D(g(T))$ such that

$$
\langle\phi \mid g(T) \psi\rangle=\int_{\mathbb{R}} g(\tau) \mathrm{d} \mu_{\phi, \psi}(\tau) \text { for all } \phi \in \mathrm{H} \text { and } \psi \in D(g(T)) .
$$

The support of $P^{(T)}$ coincides with the spectrum $\sigma(T)$, and the integration in (3) can be restricted accordingly without affecting the identity. The spectral decomposition of $T$ is nothing but (3) with $g: \mathbb{R} \ni \tau \mapsto \tau \in \mathbb{R}$ and the spectral theorem just states that this decomposition exists and $P^{(T)}$ is uniquely determined by $T$.

\section{From $\mathbb{C}^{n}$ to an infinite-dimensional Hilbert space}

\subsection{The finite-dimensional Hilbert space case}

The finite-dimensional case is a comfortable arena where building up our formalism, since no problems related to domains and choices of topologies take place. Let us therefore suppose that $\mathrm{H}$ is finite dimensional. Without loss of generality, we can assume $\mathrm{H}=\mathbb{C}^{k}$. From the elementary spectral theory [37], we define

$$
g(T):=\sum_{\tau \in \sigma(T)} g(\tau) P_{\tau}^{(T)}
$$

for every function $g: \mathbb{R} \rightarrow \mathbb{R}$ and every self-adjoint operator $T$, where $T$ is a $k \times k$ Hermitian matrix $T: \mathrm{H} \rightarrow \mathrm{H}$. The spectrum $\sigma(T) \subset \mathbb{R}$ of $T$ denotes its set of eigenvalues and $P_{\tau}^{(T)}$ indicates the orthogonal projector onto the eigenspace of $\tau \in \sigma(T)$, so that $\left\{P_{\tau}^{(T)}\right\}_{\tau \in \sigma(T)}$ completely determines the PVM $P^{(T)}$ of $T$. No technical snags pop out with the sum in (5), since $\sigma(T)$ is a non-empty set of at most $k=\operatorname{dim}(\mathrm{H})<+\infty$ elements.

We now focus on a couple of self-adjoint operators $A: \mathrm{H} \rightarrow \mathrm{H}, B: \mathrm{H} \rightarrow \mathrm{H}$ with, in general, $A B \neq B A$. We intend to write $f(a A+b B)$ in terms of the PVMs $P^{(A)}$ and $P^{(B)}$ for $a, b \in \mathbb{R}$ and where $f: \mathbb{R} \rightarrow \mathbb{C}$ is a sufficiently regular function. For technical reasons which will be evident shortly, we henceforth assume that: (i) $f: \mathbb{R} \rightarrow \mathbb{C}$ is continuous; (ii) $f$ belongs to $L^{1}(\mathbb{R}, \mathrm{d} x)$; (iii) the inverse Fourier transform $\check{f}(t):=\frac{1}{2 \pi} \int_{\mathbb{R}} f(x) \mathrm{e}^{-i t x} \mathrm{~d} x$ belongs to $L^{1}(\mathbb{R}, \mathrm{d} x)$. Every $f \in \mathscr{S}(\mathbb{R})$ in particular satisfies the said three hypotheses. Assuming (i),(ii) and (iii), the pointwise

\footnotetext{
4 Alternatively, (4) is a consequence of a different but equivalent definition of the right-hand side of (3), which uses the strong operator topology instead of the weak one. See, e.g., [25].
} 
inversion theorem of the Fourier transform is valid: $f(x)=\int_{\mathbb{R}} \check{f}(t) \mathrm{e}^{i t x} \mathrm{~d} t$ for every $x \in \mathbb{R}$.

The pivotal tool underpinning our result is the celebrated Trotter formula,

$$
\mathrm{e}^{i t(a A+b B)}=\lim _{N \rightarrow+\infty}\left(\mathrm{e}^{i \frac{t}{N} a A} \mathrm{e}^{i \frac{t}{N} a B}\right)^{N}, \quad t \in \mathbb{R},
$$

whose convergence is here referred to every normed topology on $\mathfrak{B}(\mathrm{H})$, since that (Banach) space is finite dimensional. Spectrally decomposing the exponentials on both sides into a finite linear combinations of matrices according to (5) and using of Lebesgue's dominated convergence theorem term by term while integrating both sides against $\breve{f}(t)$, we have,

$$
\int_{\mathbb{R}} \check{f}(t) \mathrm{e}^{i t(a A+b B)} \mathrm{d} t=\lim _{N \rightarrow+\infty} \int_{\mathbb{R}} \check{f}(t) \underbrace{\mathrm{e}^{i \frac{t}{N} a A} \mathrm{e}^{i \frac{t}{N} a B} \cdots \mathrm{e}^{i \frac{t}{N} a A} \mathrm{e}^{i \frac{t}{N} a B}}_{N \text { times }} \mathrm{d} t .
$$

Exploiting (5), identity (7) can be expanded as

$$
\begin{aligned}
& \sum_{\tau \in \sigma(a A+b B)}\left(\int_{\mathbb{R}} \check{f}(t) \mathrm{e}^{i t \tau} \mathrm{d} t\right) P_{\tau}^{(a A+b B)} \\
& =\lim _{N \rightarrow+\infty} \sum_{\lambda_{j} \in \sigma(A), \mu_{j} \in \sigma(B)}\left(\int_{\mathbb{R}} \check{f}(t) \mathrm{e}^{i \frac{t}{N} a \lambda_{1}} \mathrm{e}^{i \frac{t}{N} b \mu_{1}}\right. \\
& \left.\quad \cdots \mathrm{e}^{i \frac{t}{N} a \lambda_{N}} \mathrm{e}^{i \frac{t}{N} b \mu_{N}} \mathrm{~d} t\right) P_{\lambda_{1}}^{(A)} P_{\mu_{1}}^{(B)} \cdots P_{\lambda_{N}}^{(A)} P_{\mu_{N}}^{(B)} .
\end{aligned}
$$

Since $f(x)=\int_{\mathbb{R}} \check{f}(t) \mathrm{e}^{i t x} \mathrm{~d} t$ and taking (5) into account on the left-hand side, we obtain the prototype of our general formula

$$
f(a A+b B)=\lim _{N \rightarrow+\infty} \sum_{\lambda_{j} \in \sigma(A), \mu_{j} \in \sigma(B)} f\left(\frac{1}{N} \sum_{j=1}^{N}\left(a \lambda_{j}+b \mu_{j}\right)\right) P_{\lambda_{1}}^{(A)} P_{\mu_{1}}^{(B)} \cdots P_{\lambda_{N}}^{(A)} P_{\mu_{N}}^{(B)} .
$$

In $\mathrm{H}=\mathbb{C}^{k}$, it is not difficult to prove that $A B-B A=0$ implies $\left(\mathrm{e}^{i \frac{t}{N} a A} \mathrm{e}^{i \frac{t}{N} a B}\right)^{N}=$ $\mathrm{e}^{i t(a A+b B)}$. Hence, following our derivation of (8), the limit on the right-hand side of (8) is not necessary since its argument turns out to be constant in $N$. Therefore, for commuting $A$ and $B$, equation (8) reduces to

$$
f(a A+b B)=\sum_{\lambda \in \sigma(A), \mu \in \sigma(B)} f(a \lambda+b \mu) P_{\lambda}^{(A)} P_{\mu}^{(B)},
$$

which is nothing but the usual spectral decomposition of the left-hand side with respect to the joint $P V M$ of $A$ and $B$.

A physically important case of (9) would be obtained by choosing $f=1_{[\alpha, \beta)}$ (the indicator function of the set $[\alpha, \beta))$. As a matter of fact, $1_{[\alpha, \beta)}(a A+b B)=P_{[\alpha, \beta)}^{(a A+b B)}$ 
is the spectral projection of $[\alpha, \beta)$ and this family of projectors, when $\alpha<\beta$, includes the full information of the PVM of $a A+b B$. Unfortunately, such $f$ does not satisfy (i) and (iii); hence, a further regularization procedure is necessary by means of a family $1_{[\alpha, \beta)}^{(\epsilon)} \in \mathscr{S}(\mathbb{R})$ suitably converging to $1_{[\alpha, \beta)}$ as $\epsilon \rightarrow 0^{+}$. In this way, the identity

$$
\begin{aligned}
P_{[\alpha, \beta)}^{(a A+b B)}= & \lim _{\epsilon \rightarrow 0^{+}} \lim _{N \rightarrow+\infty} \sum_{\lambda_{j} \in \sigma(A), \mu_{j} \in \sigma(B)} 1_{[\alpha, \beta)}^{(\epsilon)} \\
& \left(\frac{1}{N} \sum_{j=1}^{N}\left(a \lambda_{j}+b \mu_{j}\right)\right) P_{\lambda_{1}}^{(A)} P_{\mu_{1}}^{(B)} \cdots P_{\lambda_{N}}^{(A)} P_{\mu_{N}}^{(B)}
\end{aligned}
$$

can be established. We shall prove it later in a much more general context as identity (38).

The requirement $f, \check{f} \in L^{2}(\mathbb{R}, \mathrm{d} x)$ can be relaxed making stronger the regularity requirement on $f$ as a consequence of the following argument. If $f, g$ satisfy (i), (ii), (ii) and also $f(x)=g(x)$ for $x \in[-\|a A\|-\|b B\|,\|a A\|+\|b B\|]$, where the norm is the operator norm, then the well-known general estimate for self-adjoint operators that $\sigma(T) \subset[-\|T\|,\|T\|]$, yields both

$$
f(a A+b B)=g(a A+b B)
$$

from (5), and

$$
f\left(\frac{1}{N} \sum_{j=1}^{N}\left(\lambda_{j}+\mu_{j}\right)\right)=g\left(\frac{1}{N} \sum_{j=1}^{N}\left(\lambda_{j}+\mu_{j}\right)\right) \text { for } \lambda_{j} \in \sigma(A), \mu_{j} \in \sigma(B) .
$$

It is consequently safe to extend the validity of (8) to every complex valued $f \in C^{\infty}(\mathbb{R})$ simply smoothly changing $f$ to a function of $\mathscr{S}(\mathbb{R})$ outside the interval $[-\|a A\|-$ $\|b B\|,\|a A\|+\|b B\|]$. In particular, $f$ can be chosen as a polynomial of a single real variable. In this way, we also obtain the elementary Jordan-algebra operations on $A$ and $B$ written in terms of the PVMs of $A$ and $B$ as the second identity below. If $a, b \in \mathbb{R}$,

$$
a A+b B=\lim _{N \rightarrow+\infty} \sum_{\lambda_{j} \in \sigma(A), \mu_{j} \in \sigma(B)}\left(a \sum_{j=1}^{N} \frac{\lambda_{j}}{N}+b \sum_{j=1}^{N} \frac{\mu_{j}}{N}\right) P_{\lambda_{1}}^{(A)} P_{\mu_{1}}^{(B)} \cdots P_{\lambda_{N}}^{(A)} P_{\mu_{N}}^{(B)}
$$

and

$$
\frac{1}{2}(A B+B A)=\lim _{N \rightarrow+\infty} \sum_{\lambda_{j} \in \sigma(A), \mu_{j} \in \sigma(B)}\left(\sum_{j=1}^{N} \frac{\lambda_{j}}{N}\right)\left(\sum_{j=1}^{N} \frac{\mu_{j}}{N}\right) P_{\lambda_{1}}^{(A)} P_{\mu_{1}}^{(B)} \cdots P_{\lambda_{N}}^{(A)} P_{\mu_{N}}^{(B)}
$$


The former is simply obtained from (8) choosing $f(s)=s$. The latter arises from a more involved though elementary procedure. The left-hand side of (13) can be written as

$$
\frac{1}{2}\left[(A+B)^{2}-A^{2}-B^{2}\right]=f(a A+b B)+g\left(a^{\prime} A+b^{\prime} B\right)+g\left(a^{\prime \prime} A+b^{\prime \prime} B\right)
$$

where $f(s):=s^{2}, g(s)=-s^{2}, a=b=1, a^{\prime}=1, b^{\prime}=0, a^{\prime \prime}=0, b^{\prime \prime}=1$. Linearity in $f$ of the right-hand side of (8) (also with different constants $a, b$ ) immediately gives rise to (13). A more detailed discussion will appear in Sect. 3, where (13) is extended to polynomials (Theorem 3.6).

With an argument similar to the one leading to (11) and using the fact that $\sigma(a A+$ $b B)$ is a finite discrete set of reals, we achieve another useful result. If $\beta \notin \sigma(a A+b B)$, identity (10) can be alternatively written

$$
P_{[\alpha, \beta)}^{(a A+b B)}=\lim _{N \rightarrow+\infty} \sum_{\substack{\lambda_{j} \in \sigma(A) \\ \mu_{j} \in \sigma(B)}} 1_{[\alpha, \beta)}^{\prime}\left(\frac{1}{N} \sum_{j=1}^{N}\left(a \lambda_{j}+b \mu_{j}\right)\right) P_{\lambda_{1}}^{(A)} P_{\mu_{1}}^{(B)} \cdots P_{\lambda_{N}}^{(A)} P_{\mu_{N}}^{(B)},
$$

where $1_{[\alpha, \beta)}^{\prime} \in \mathcal{D}(\mathbb{R})$ is a map which attains the constant value 1 over $[\alpha, \beta)$ and smoothly vanishes on $\sigma(a A+b B) \backslash[\alpha, \beta)$.

When trying to extend (8) to the case of a general (separable) Hilbert space $\mathrm{H}$ for generally unbounded self-adjoint operators $A$ and $B$, several evident technical issues arise. First of all, usual domain problems have to be fixed. These problems are tantamount to corresponding domain issues with Trotter's formula which are well known and definitely fixed [33, Theorem VIII.31]. A sufficiently general setup consists of assuming that $a A+b B$ is essentially self-adjoint over its natural domain $D(A) \cap$ $D(B)$ (if $a b \neq 0$ ). A much harder problem is the interpretation of the operator-valued integral appearing in the right-hand side of (8), especially in case the spectrum of either $A$ or $B$ includes a continuous part. Actually, problems of a similar nature arise also when $A$ and $B$ have pure point spectra, but $\mathrm{H}$ is infinite dimensional. In the following sections, we will address those technical problems and other related ones, ending up with a wide generalization of (8)—c.f. Theorem 2.5 .

\subsection{The unital Banach algebra of complex Borel measures}

Before entering the technical details of the core of the paper, it is necessary to introduce the space of functions we will use on both sides of the extension of (8).

Definition 2.1 The space of complex measures on $\mathbb{R}$ denoted by $\mathscr{M}(\mathbb{R})$ is the complex linear space of $\mathbb{C}$-valued $\sigma$-additive maps $v: \mathscr{B}(\mathbb{R}) \rightarrow \mathbb{C}$. The space of Fourier transforms of complex measures on $\mathbb{R}$ consists of the complex linear space $\mathscr{F}(\mathbb{R})$ of functions of the form

$$
f_{v}(x):=\int_{\mathbb{R}} \mathrm{e}^{i x y} \mathrm{~d} v(y), \quad x \in \mathbb{R},
$$


for some $v \in \mathscr{M}(\mathbb{R})$.

The linear map

$$
F: \mathscr{M}(\mathbb{R}) \ni \nu \mapsto f_{v} \in \mathscr{F}(\mathbb{R})
$$

is injective (this is a straightforward extension of [5, Theorem 26.2]) so that it defines a linear isomorphism. From the standard properties of Fourier transform, it is not difficult to prove that $\mathscr{F}$ includes the maps satisfying (i), (ii), (iii) we used in Sect. 2.1. Furthermore,

$$
\mathscr{F}(\mathbb{R}) \subset C_{b}(\mathbb{R}), \quad \mathscr{S}(\mathbb{R}) \subset \mathscr{F}(\mathbb{R}) \subset \mathscr{S}^{\prime}(\mathbb{R}), \quad \mathcal{D}(\mathbb{R}) \subset \mathscr{F}(\mathbb{R}) \subset \mathcal{D}^{\prime}(\mathbb{R})
$$

Above, $C_{b}(\mathbb{R})$ is the commutative unital Banach algebra (with norm $\|\cdot\|_{\infty}$ ) of bounded continuous complex-valued functions over $\mathbb{R}, \mathscr{S}(\mathbb{R})$ and $\mathcal{D}(\mathbb{R})$ are, respectively, the space of Schwartz (complex) functions and the space of the (complex) test functions over $\mathbb{R}$, and $\mathscr{S}^{\prime}(\mathbb{R})$ and $\mathcal{D}^{\prime}(\mathbb{R})$ denote the associated spaces of distributions.

The linear space $\mathscr{M}(\mathbb{R})$ turns out to be a commutative unital Banach algebra where (a) the product of two measures $v$ and $v^{\prime}$ is their convolution $v * v^{\prime}$, (b) the unit is the Dirac point mass $\delta_{0}$ concentrated at 0 and (c) the norm of a measure $v$ is defined as its total variation $\|v\|=|v|(\mathbb{R}) . \mathscr{M}(\mathbb{R})$ also admits a norm-preserving unit-preserving antilinear involution given by the complex conjugation of measure $v^{*}:=\bar{\nu}$. The linear isomorphism (16) induces a unital commutative Banach algebra structure over $\mathscr{F}(\mathbb{R})$ when defining $\left\|f_{v}\right\|_{\mathscr{F}}:=\|v\|$. More precisely, the map $F$ promoted to an isomorphism of Banach algebras transforms the convolution of measures into the pointwise product of corresponding functions and the unit of $\mathscr{M}(\mathbb{R})$ to the constant function 1 . The involution of $\mathscr{M}(\mathbb{R})$ becomes the norm-preserving unit-preserving antilinear involution over $\mathscr{F}(\mathbb{R})$, given by $f^{*}(x):=\overline{f(-x)}$, the bar denoting the complex conjugation. The definition of $\|f\|_{\mathscr{F}}$ easily implies

$$
\|f\|_{\infty} \leq\|f\|_{\mathscr{F}}, \quad f \in \mathscr{F}(\mathbb{R})
$$

If $f \in \mathscr{S}(\mathbb{R})\left(\right.$ or $\mathcal{D}(\mathbb{R})$ ), then $f=f_{v}$ where $\mathrm{d} v=(2 \pi)^{-1} \check{f} \mathrm{~d} x$ and $\check{f}(y)=$ $\int_{\mathbb{R}} \mathrm{e}^{-i x y} f(x) \mathrm{d} y$ is the Fourier anti-transform of $f$. In this case

$$
\|f\|_{\mathscr{F}}=(2 \pi)^{-1}\|\check{f}\|_{L^{1}(\mathbb{R}, \mathrm{d} x)}, \quad f \in \mathscr{S}(\mathbb{R})
$$

\subsection{Regularized products of non-commuting PVMs of self-adjoint operators}

This section and the subsequent one are devoted to extend formula (8) to the case of an infinite-dimensional, though separable, Hilbert space $\mathrm{H}$. We will suppose that the operators $A$ and $B$ are unbounded self-adjoint operators with domains $D(A)$ and $D(B)$, respectively, and that their linear combination $a A+b B$, for suitable $a, b \in \mathbb{R}$, is essentially self-adjoint on its standard domain according to Sect. 1.

In other words, we want to construct a functional calculus and the spectral measure of the self-adjoint operator given by the closure $\overline{a A+b B}$ out of the PVMs $P^{(A)}, P^{(B)}$ 
of $A$ and $B$, respectively, proving the following suggestive formula if $\psi \in \mathrm{H}$ and $f \in \mathscr{F}(\mathbb{R}):$

$$
f(\overline{a A+b B}) \psi=\lim _{N \rightarrow+\infty} \int_{\mathbb{R}^{2 N}} f\left(\frac{1}{N} \sum_{n=1}^{N}\left(a \lambda_{n}+b \mu_{n}\right)\right) \mathrm{d} P_{\lambda_{1}}^{(A)} \mathrm{d} P_{\mu_{1}}^{(B)} \cdots \mathrm{d} P_{\lambda_{N}}^{(A)} \mathrm{d} P_{\mu_{N}}^{(B)} \psi
$$

The operator $f(\overline{a A+b B})$ on the left-hand side is independently defined by (3) and its domain is the whole $\mathrm{H}$ according to (3) since $f$ is bounded.

As a first step, we address the problem of the interpretation of the operators

$$
\int_{\mathbb{R}^{2 N}} f\left(\frac{1}{N} \sum_{n=1}^{N}\left(a \lambda_{n}+b \mu_{n}\right)\right) \mathrm{d} P_{\lambda_{1}}^{(A)} \mathrm{d} P_{\mu_{1}}^{(B)} \cdots \mathrm{d} P_{\lambda_{N}}^{(A)} \mathrm{d} P_{\mu_{N}}^{(B)}
$$

after the symbol of limit on the right-hand side of (19). In fact, we cannot generally interpret the integration appearing in (20) like the one in (9), i.e., as referred to the joint $P V M$ of $N$ copies of $P^{(A)}$ and $P^{(B)}$ (see, e.g., [25,34]). This is because we are focusing on the generic situation where $P^{(A)}$ and $P^{(B)}$ do not commute. In the general case, operators (20) are defined out of a regularized natural quadratic form we are going to introduce with the following crucial technical result. We stress that we will not use the hypothesis of essential self-adjointness of $a A+b B$ at this stage of the construction.

Lemma 2.2 Let $A$ and $B$ be a pair of self-adjoint operators over the separable Hilbert space $H$ as in (3), and $Q:=\left\{Q_{n}\right\}_{n \in \mathbb{N}}$ a sequence of orthogonal projectors over respective finite-dimensional subspaces such that $Q_{n} \rightarrow I$ strongly as $n \rightarrow+\infty$. If $\phi, \psi \in H$ and $N \in \mathbb{N}$, then the following facts are valid.

(a) For every $n \in \mathbb{N}$, there is a unique complex Borel measure $v_{\phi, \psi, Q}^{(N, n)}$ over $\mathbb{R}^{2 N}$ such that

$$
v_{\phi, \psi, Q}^{(N, n)}\left(\times_{\ell=1}^{N} I_{\ell} \times J_{\ell}\right):=\left\langle\phi \mid P_{I_{1}}^{(A)} Q_{n} P_{J_{1}}^{(B)} \cdots P_{I_{N}}^{(A)} Q_{n} P_{J_{N}}^{(B)} \psi\right\rangle,
$$

with $I_{\ell}, J_{\ell}$ arbitrary Borel sets in $\mathbb{R}$. The support of the measure satisfies

$$
\operatorname{supp}\left(\left|v_{\phi, \psi, Q}^{(N, n)}\right|\right) \subset \underbrace{\sigma(A) \times \sigma(B) \times \cdots \times \sigma(A) \times \sigma(B)}_{2 N \text { times }} .
$$

(b) For every $f \in \mathscr{F}(\mathbb{R})$ and, independently of the choice of the sequence $\left\{Q_{n}\right\}_{n \in \mathbb{N}}$, the following limit holds for $a, b \in \mathbb{R}$ :

$$
\begin{aligned}
& \lim _{n \rightarrow \infty} \int_{\mathbb{R}^{2 N}} f\left(\frac{1}{N} \sum_{l=1}^{N}\left(a \lambda_{l}+b \mu_{l}\right)\right) \mathrm{d} v_{\phi, \psi, Q}^{(N, n)}\left(\lambda_{1}, \mu_{1}, \ldots, \lambda_{N}, \mu_{N}\right) \\
& =\int_{\mathbb{R}}\left\langle\phi \mid\left(e^{i \frac{t}{N} a A} e^{i \frac{t}{N} b B}\right)^{N} \psi\right\rangle \mathrm{d} \nu_{f}(t),
\end{aligned}
$$

with $v_{f}:=F^{-1}(f)$ according to (15-16). 
Proof See "1"

Analogously to the use of (4) to define the right-hand side in (3), the idea is now to define the operator in (20) in order that the associated quadratic form coincides with the limit of the regularized sequences as on the left-hand side of (23).

Theorem 2.3 Let A and B be a pair of self-adjoint operators over the separable Hilbert space $H$ as in (3), $f \in \mathscr{F}$, and $N \in \mathbb{N}$. For $a, b \in \mathbb{R}$, there is a unique operator, denoted by

$$
\int_{\mathbb{R}^{2 N}} f\left(\frac{1}{N} \sum_{n=1}^{N}\left(a \lambda_{n}+b \mu_{n}\right)\right) d P_{\lambda_{1}}^{(A)} d P_{\mu_{1}}^{(B)} \cdots d P_{\lambda_{N}}^{(A)} d P_{\mu_{N}}^{(B)} \in \mathfrak{B}(H),
$$

such that

$$
\begin{aligned}
& \left\langle\phi \mid \int_{\mathbb{R}^{2 N}} f\left(\frac{1}{N} \sum_{n=1}^{N}\left(a \lambda_{n}+b \mu_{n}\right)\right) d P_{\lambda_{1}}^{(A)} d P_{\mu_{1}}^{(B)} \cdots d P_{\lambda_{N}}^{(A)} d P_{\mu_{N}}^{(B)} \psi\right\rangle \\
& =\lim _{n \rightarrow \infty} \int_{\mathbb{R}^{2 N}} f\left(\frac{1}{N} \sum_{l=1}^{N}\left(a \lambda_{l}+b \mu_{l}\right)\right) \mathrm{d} \nu_{\phi, \psi, Q}^{(N, n)}\left(\lambda_{1}, \mu_{1}, \ldots, \lambda_{N}, \mu_{N}\right),
\end{aligned}
$$

if $\phi, \psi \in H$.

Above, for every $n \in \mathbb{N}$, the complex Borel measure $v_{\phi, \psi, Q}^{(N, n)}$ is uniquely defined by

$$
v_{\phi, \psi, Q}^{(N, n)}\left(\times_{\ell=1}^{N} I_{\ell} \times J_{\ell}\right):=\left\langle\phi \mid P_{I_{1}}^{(A)} Q_{n} P_{J_{1}}^{(B)} \cdots P_{I_{N}}^{(A)} Q_{n} P_{J_{N}}^{(B)} \psi\right\rangle \text { with } I_{l}, J_{l} \in \mathscr{B}(\mathbb{R})
$$

for a sequence of finite-dimensional orthogonal projectors $Q:=\left\{Q_{n}\right\}_{n \in \mathbb{N}}$ with $Q_{n} \rightarrow$ I strongly as $n \rightarrow+\infty$. The limit in (25) is, however, independent of the choice of $\left\{Q_{n}\right\}_{n \in \mathbb{N}}$.

The following further facts are true.

(i) The map

$$
\mathscr{F} \ni f \mapsto \int_{\mathbb{R}^{2 N}} f\left(\frac{1}{N} \sum_{n=1}^{N}\left(a \lambda_{n}+b \mu_{n}\right)\right) d P_{\lambda_{1}}^{(A)} d P_{\mu_{1}}^{(B)} \cdots d P_{\lambda_{N}}^{(A)} d P_{\mu_{N}}^{(B)} \in \mathfrak{B}(H)
$$

is linear.

(ii) The following inequality holds

$$
\left\|\int_{\mathbb{R}^{2 N}} f\left(\frac{1}{N} \sum_{n=1}^{N}\left(a \lambda_{n}+b \mu_{n}\right)\right) d P_{\lambda_{1}}^{(A)} d P_{\mu_{1}}^{(B)} \cdots d P_{\lambda_{N}}^{(A)} d P_{\mu_{N}}^{(B)}\right\| \leq\|f\|_{\mathscr{F}} .
$$


(iii) If $\phi, \psi \in H$, the restricted maps

$$
\begin{aligned}
& \mathscr{S}(\mathbb{R}) \ni f \mapsto\left\langle\phi \mid \int_{\mathbb{R}^{2 N}} f\left(\frac{1}{N} \sum_{n=1}^{N}\left(a \lambda_{n}+b \mu_{n}\right)\right) d P_{\lambda_{1}}^{(A)} d P_{\mu_{1}}^{(B)} \cdots d P_{\lambda_{N}}^{(A)} d P_{\mu_{N}}^{(B)} \psi\right\rangle \\
& \mathscr{S}(\mathbb{R}) \ni f \mapsto \int_{\mathbb{R}^{2 N}} f\left(\frac{1}{N} \sum_{n=1}^{N}\left(a \lambda_{n}+b \mu_{n}\right)\right) d P_{\lambda_{1}}^{(A)} d P_{\mu_{1}}^{(B)} \cdots d P_{\lambda_{N}}^{(A)} d P_{\mu_{N}}^{(B)}
\end{aligned}
$$

are, respectively, a Schwartz distribution and a $\mathscr{B}(H)$-valued Schwartz distribution. The same facts hold if replacing $\mathscr{S}(\mathbb{R})$ with $\mathcal{D}(\mathbb{R})$ and $\mathscr{S}^{\prime}(\mathbb{R})$ with $\mathcal{D}^{\prime}(\mathbb{R})$.

(iv) If $A, B \in \mathfrak{B}(H)$ and $\operatorname{supp}(f) \cap[-\|a A\|-\|b B\|$, $\|a A\|+\|b B\|]=\varnothing$, then

$$
\int_{\mathbb{R}^{2 N}} f\left(\frac{1}{N} \sum_{n=1}^{N}\left(a \lambda_{n}+b \mu_{n}\right)\right) d P_{\lambda_{1}}^{(A)} d P_{\mu_{1}}^{(B)} \cdots d P_{\lambda_{N}}^{(A)} d P_{\mu_{N}}^{(B)}=0 .
$$

(v) If $\bar{z}$ denotes the complex conjugate of $z \in \mathbb{C}$, then

$$
\begin{aligned}
& {\left[\int_{\mathbb{R}^{2 N}} f\left(\frac{1}{N} \sum_{n=1}^{N}\left(a \lambda_{n}+b \mu_{n}\right)\right) d P_{\lambda_{1}}^{(A)} d P_{\mu_{1}}^{(B)} \cdots d P_{\lambda_{N}}^{(A)} d P_{\mu_{N}}^{(B)}\right]^{*}} \\
& =\int_{\mathbb{R}^{2 N}} f\left(\frac{1}{N} \sum_{n=1}^{N}\left(a \lambda_{n}+b \mu_{n}\right)\right) d P_{\mu_{1}}^{(B)} d P_{\lambda_{1}}^{(A)} \cdots d P_{\mu_{N}}^{(B)} d P_{\lambda_{N}}^{(A)}
\end{aligned}
$$

Proof If $v_{f}:=F^{-1}(f)$ according to (15)-(16), the map appearing on the right-hand side of (23), that is,

$$
\mathrm{H} \times \mathrm{H} \times \mathscr{F} \ni(\phi, \psi, f) \mapsto I_{\phi, \psi}^{(N)}(f):=\int_{\mathbb{R}}\left\langle\phi \mid\left(\mathrm{e}^{i \frac{t}{N} a A} \mathrm{e}^{i \frac{t}{N} b B}\right)^{N} \psi\right\rangle \mathrm{d} \nu_{f}(t),
$$

is by construction linear in $f$ and $\psi$, antilinear in $\phi$ and satisfies

$$
\left|I_{\phi, \psi}^{(N)}(f)\right| \leq\|\phi\|_{H}\|\psi\|_{H}\|f\|_{\mathscr{F}} .
$$

As a consequence, identity (23) permits us to exploit Riesz' lemma defining the wanted operator as required and independently from the regularizing sequence $\left\{Q_{n}\right\}_{n \in \mathbb{N}}$. Indeed, for any $\psi, f$, the map $\phi \mapsto I_{\phi, \psi}^{(N)}(f)$ defines a bounded antilinear functional, hence Riesz representation theorem allows to write the following identity

$$
I_{\phi, \psi}^{(N)}(f)=\left\langle\phi, \xi_{f}\right\rangle
$$


for a suitable vector $\xi_{f} \in \mathrm{H}$ such that

$$
\left\|\xi_{f}\right\| \leq\|\psi\|_{H}\|f\|_{\mathscr{F}} .
$$

The linear dependence of $I_{\phi, \psi}^{(N)}(f)$ on $\psi$ yields the linearity of the map $\psi \mapsto \xi_{\psi}:=$ $L(\psi)$ as well. Finally, inequality (31) allows to prove that the operator $L$ is bounded and by definition it coincides with (24). Linearity of $f \mapsto I_{\phi, \psi}^{(N)}(f)$ and (30) prove (i) and (ii).

Regarding (iii), we observe that from the continuity properties of the Fourier transform, $\mathscr{S}(\mathbb{R}) \ni f_{n} \rightarrow 0$ in the $\mathscr{S}$-topology (as $n \rightarrow+\infty$ ) implies that the sequence of anti-transforms $\breve{f}_{n}$ vanishes in the same topology, so that, in particular, $\left\|\breve{f}_{n}\right\|_{L^{1}(\mathbb{R}, \mathrm{d} x)} \rightarrow 0$. Since $\left\|f_{n}\right\|_{\mathscr{F}}=(2 \pi)^{-1}\left\|\check{f}_{n}\right\|_{L^{1}(\mathbb{R}, \mathrm{d} x)}$ from (18), we have that $\left\|f_{n}\right\| \mathscr{F} \rightarrow 0$. The linear maps (27) and (28) are therefore $\mathscr{S}$-continuous in view of inequality (26) so that they are Schwartz distributions by definition. Since Schwartz distributions are distributions over $\mathcal{D}(\mathbb{R})$, the result is also valid when restricting our map to $\mathcal{D}(\mathbb{R})$.

(iv) First observe that $\sigma(A) \subset[-\|A\|,\|A\|]$ and $\sigma(B) \subset[-\|B\|,\|B\|]$. At this juncture, inclusion (22) and definition (25) imply that the integration over $\mathbb{R}^{2 N}$ on the right-hand side of (25) can actually be restricted to the set determined by imposing $\lambda_{j} \in[-\|A\|,\|A\|]$ and $\mu_{j} \in[-\|B\|, \mid B \|]$ for $j=1, \ldots, N$, without affecting the final result. If the support of $f$ is disjoint from $[-\|a A\|-\|b B\|,\|a A\|+\|b B\|]$, then $f\left(\frac{1}{N} \sum_{j=1}^{N}\left(a \lambda_{j}+b \mu_{j}\right)\right)$ vanishes when $\lambda_{j} \in[-\|A\|,\|A\|]$ and $\mu_{j} \in[-\|B\|,\|B\|]$ so that the integral in (25) vanishes as well for every $n$, producing 0 as limit for $n \rightarrow+\infty$.

The proof of (v) follows at once from the definitions.

Remark 2.4 (1) If $\mathrm{H}$ is finite dimensional, all $Q_{n}$ on the right-hand side of (21) can be removed, simply choosing $Q_{n}=I$, finding the formulas achieved in Sect. 2.1. For infinite-dimensional $\mathrm{H}$, there are cases where no complex measure satisfying identity (21) exists if $Q_{n}=I$ for all $n-$ c.f. Sect. 4.1.

(2) The existence of sequences $\left\{Q_{n}\right\}_{n \in \mathbb{N}}$ strongly approximating the identity operator with finite-dimensional projection spaces is equivalent to separability of $\mathrm{H}$. Hence, separability hypothesis cannot be relaxed.

\subsection{Main result}

We have reached a position to establish our main theorem when, in addition to the already assumed hypotheses on $A$ and $B$, we use the requirement that $a A+b B$ is essentially self-adjoint.

Theorem 2.5 Let $A, B$ be self-adjoint operators on a separable Hilbert space, $a, b \in \mathbb{R}$ and let us assume that $a A+b B$ is essentially self-adjoint on $D(a A) \cap D(b B)$. Then, the following facts hold for $f \in \mathscr{F}(\mathbb{R})$. 
(a) Denote the strong operator limit by $s-\lim$. Then,

$$
f(\overline{a A+b B})=s^{-} \lim _{N \rightarrow+\infty} \int_{\mathbb{R}^{2 N}} f\left(\frac{1}{N} \sum_{n=1}^{N}\left(a \lambda_{n}+b \mu_{n}\right)\right) d P_{\lambda_{1}}^{(A)} d P_{\mu_{1}}^{(B)} \cdots d P_{\lambda_{N}}^{(A)} d P_{\mu_{N}}^{(B)},
$$

and a similar identity is valid if, keeping the left-hand side, we swap $d P_{\lambda_{j}}^{(A)}$ and $d P_{\mu_{j}}^{(B)}$ for $j=1,2, \ldots, N$ on the right-hand side.

(b) If the PVMs $P^{(a A)}$ and $P^{(b B)}$ commute (this fact holds in particular if either $a=0$ or $b=0)$, then

$$
f(\overline{a A+b B})=\int_{\mathbb{R}^{2 N}} f\left(\frac{1}{N} \sum_{n=1}^{N}\left(a \lambda_{n}+b \mu_{n}\right)\right) d P_{\lambda_{1}}^{(A)} d P_{\mu_{1}}^{(B)} \cdots d P_{\lambda_{N}}^{(A)} d P_{\mu_{N}}^{(B)}
$$

for every $N \in \mathbb{N}$.

(c) If the PVMs $P^{(A)}$ and $P^{(B)}$ commute, then

$$
v_{\phi, \psi, Q}^{(1, n)}(I \times J)=\left\langle\phi \mid P_{I}^{(A)} Q_{n} P_{J}^{(B)} \psi\right\rangle \rightarrow\left\langle\phi \mid P_{I \times J}^{(A, B)} \psi\right\rangle \text { as } n \rightarrow+\infty,
$$

where $P^{(A, B)}: \mathscr{B}\left(\mathbb{R}^{2}\right) \rightarrow \mathscr{L}(H)$ is the joint $P V M$ of $A$ and $B$ and the right-hand side of (33)—defined as in (25) — with $N=1$ can be interpreted as an integration with respect to that joint $P V M$.

Proof (a) From now on, $v_{f}:=F^{-1}(f)$ according to (15)-(16). For $N \in \mathbb{N}$, we define the operator

$$
\int_{\mathbb{R}}\left(\mathrm{e}^{i \frac{t}{N} a A} \mathrm{e}^{i \frac{t}{N} b B}\right)^{N} \mathrm{~d} \nu_{f}(t)
$$

as the unique operator $S \in \mathfrak{B}(\mathrm{H})$ such that

$$
\langle\phi \mid S \psi\rangle=\int_{\mathbb{R}}\left\langle\phi \mid\left(\mathrm{e}^{i \frac{t}{N} a A} \mathrm{e}^{i \frac{t}{N} b B}\right)^{N} \psi\right\rangle \mathrm{d} v_{f}(t) \quad \text { if } \phi, \psi \in \mathrm{H} .
$$

The operator

$$
\int_{\mathbb{R}} \mathrm{e}^{i t(\overline{a A+b B})} \mathrm{d} v_{f}(t)
$$

is analogously defined.

The former operator is already known. Indeed, from (23) and (25), we find

$$
\begin{aligned}
& \int_{\mathbb{R}}\left(\mathrm{e}^{i \frac{t}{N} a A} \mathrm{e}^{i \frac{t}{N} b B}\right)^{N} \mathrm{~d} v_{f}(t) \\
& =\int_{\mathbb{R}^{2 N}} f\left(\frac{1}{N} \sum_{n=1}^{N}\left(a \lambda_{n}+b \mu_{n}\right)\right) \mathrm{d} P_{\lambda_{1}}^{(A)} \mathrm{d} P_{\mu_{1}}^{(B)} \cdots \mathrm{d} P_{\lambda_{N}}^{(A)} \mathrm{d} P_{\mu_{N}}^{(B)} .
\end{aligned}
$$


We now turn our attention to the operator in (34). Let $P^{(\overline{a A+b B})}$ be the spectral measure of the self-adjoint operator $\overline{a A+b B}$ and set $\mu_{\phi, \psi}(E)=\left\langle\phi \mid P^{(\overline{a A+b B})}(E) \psi\right\rangle, E \in$ $\mathscr{B}(\mathbb{R})$, the complex Borel measure associated with $\phi$ and $\psi$. By standard functional calculus, by writing $f \in \mathscr{F}$ as in (15) and exploiting Fubini's theorem (all measures are finite and the integrand is bounded), we obtain:

$$
\begin{aligned}
\langle\phi \mid f(\overline{a A+b B}) \psi\rangle & =\int_{\mathbb{R}} f(\lambda) \mathrm{d} \mu_{\phi, \psi}(\lambda)=\int_{\mathbb{R}} \int_{\mathbb{R}} \mathrm{e}^{i \lambda t} \mathrm{~d} \nu_{f}(t) \mathrm{d} \mu_{\phi, \psi}(\lambda) \\
& =\int_{\mathbb{R}} \int_{\mathbb{R}} \mathrm{e}^{i \lambda t} \mathrm{~d} \mu_{\phi, \psi}(\lambda) \mathrm{d} \nu_{f}(t) \\
& =\int_{\mathbb{R}}\left\langle\phi \mid \mathrm{e}^{i t(\overline{A+B})} \psi\right\rangle \mathrm{d} \nu_{f}(t) .
\end{aligned}
$$

In other words,

$$
f(\overline{a A+b B})=\int_{\mathbb{R}} \mathrm{e}^{i t(\overline{a A+b B})} \mathrm{d} v_{f}(t) .
$$

Taking advantage of (35) and (36), the thesis of the theorem can be rephrased to

$$
\left\|\int_{\mathbb{R}} \mathrm{e}^{i t(\overline{a A+b B})} \mathrm{d} v_{f}(t) \psi-\int_{\mathbb{R}}\left(\mathrm{e}^{i \frac{t}{N} a A} \mathrm{e}^{i \frac{t}{N} b B}\right)^{N} \mathrm{~d} v_{f}(t) \psi\right\|^{2} \rightarrow 0 \text { for } N \rightarrow+\infty \text { if } \psi \in \mathbf{H} .
$$

Representing the left-hand side as an inner product and expanding it, taking the previous definitions into account, we find that the squared norm above equals

$$
\begin{gathered}
\int_{\mathbb{R}}\left(\int_{\mathbb{R}}\left\langle\mathrm{e}^{i t(\overline{a A+b B})} \psi-\left(\mathrm{e}^{i \frac{t}{N} a A} \mathrm{e}^{i \frac{t}{N} b B}\right)^{N} \psi\right| \mathrm{e}^{i s(\overline{a A+b B})} \psi\right. \\
\left.\left.-\left(\mathrm{e}^{i \frac{s}{N} a A} \mathrm{e}^{i \frac{s}{N} b B}\right)^{N} \psi\right\rangle \mathrm{d} \overline{\nu_{f}}(t)\right) \mathrm{d} \nu_{f}(s)
\end{gathered}
$$

Fubini's theorem permits us to rewrite the above integral as

$$
\begin{gathered}
\int_{\mathbb{R}^{2}}\left\langle\mathrm{e}^{i t(\overline{a A+b B})} \psi-\left(\mathrm{e}^{i \frac{t}{N} a A} \mathrm{e}^{i \frac{t}{N} b B}\right)^{N} \psi\right| \mathrm{e}^{i s(\overline{a A+b B})} \psi \\
\left.-\left(\mathrm{e}^{i \frac{s}{N} a A} \mathrm{e}^{i \frac{s}{N} b B}\right)^{N} \psi\right\rangle \mathrm{d} \overline{\nu_{f}}(t) \otimes \mathrm{d} \nu_{f}(s),
\end{gathered}
$$

where $\mathrm{d} \overline{\nu_{f}}(t) \otimes \mathrm{d} v_{f}(s)$ is the product measure. By the Trotter product formula [33, Theorem VIII.31], which is valid in our hypotheses on $a A$ and $b B$, both entries of the scalar product vanish as $N \rightarrow+\infty$. This fact implies that the integral itself vanishes as a consequence of Lebesgue's dominated convergence theorem, since the product measure is finite and the integrand is uniformly bounded in $(t, s)$ as the involved operators are unitary. In summary, (37) is valid and the proof of (a) ends since the last statement is an obvious consequence of $\overline{a A+b B}=\overline{b B+a A}$. 
(b) Exploiting the structure of the proof of (a), the thesis is valid if, for every $t \in \mathbb{R}$,

$$
\mathrm{e}^{i t(\overline{a A+b B})}=\left(\mathrm{e}^{i \frac{t}{N} a A} \mathrm{e}^{i \frac{t}{N} b B}\right)^{N}
$$

Let us prove that this identity is in fact true. Since $P^{(a A)}$ and $P^{(b B)}$ commute, referring to their joint PVM $P$, we have

$$
\mathrm{e}^{i \frac{t}{N} a A}=\int_{\mathbb{R}^{2}} \mathrm{e}^{i \frac{t}{N} \lambda} \mathrm{d} P(\lambda, \mu), \quad \mathrm{e}^{i \frac{t}{N} b B}=\int_{\mathbb{R}^{2}} \mathrm{e}^{i \frac{t}{N} \mu} \mathrm{d} P(\lambda, \mu) .
$$

From the general properties of the integral of bounded functions with respect to a given PVM,

$$
\left(\mathrm{e}^{i \frac{t}{N} a A} \mathrm{e}^{i \frac{t}{N} b B}\right)^{N}=\int_{\mathbb{R}^{2}}\left(\mathrm{e}^{i \frac{t}{N} \lambda} \mathrm{e}^{i \frac{t}{N} \mu}\right)^{N} \mathrm{~d} P(\lambda, \mu)=\int_{\mathbb{R}^{2}} \mathrm{e}^{i t \lambda} \mathrm{e}^{i t \mu} \mathrm{d} P(\lambda, \mu)=\mathrm{e}^{i t a A} \mathrm{e}^{i t b B} .
$$

Taking the strong limit as $N \rightarrow+\infty$, Trotter's formula yields

$$
\mathrm{e}^{i t(\overline{a A+b B})}=\mathrm{e}^{i t a A} \mathrm{e}^{i t b B}
$$

so that, using again $\left(\mathrm{e}^{i \frac{t}{N} a A} \mathrm{e}^{i \frac{t}{N} b B}\right)^{N}=\mathrm{e}^{i t a A} \mathrm{e}^{i t b B}$, we find

$$
\mathrm{e}^{i t(\overline{a A+b B})}=\left(\mathrm{e}^{i \frac{t}{N} a A} \mathrm{e}^{i \frac{t}{N} b B}\right)^{N}
$$

as wanted.

(c) is evident from (b), the requirement that $Q_{n} \rightarrow I$ strongly, the definition of joint PVM, and its elementary properties.

\section{Some applications}

\subsection{The PVM of $\overline{a A+b B}$ from $P^{(A)}$ and $P^{(B)}$}

As an application of Theorem 2.5, we prove that knowledge of the PVMs $P^{(A)}, P^{(B)}$ of $A$ and $B$ suffices to determine the PVM of $\overline{a A+b B}$ among all PVMs over $\mathbb{R}$. We take advantage of the well-known result that ${ }^{5} P^{(\overline{a A+b B})}$ is known once the projectors $P_{[\alpha, \beta)}^{(\overline{a A+b B})}$ are known for all $\alpha, \beta \in \mathbb{R}$ with $\alpha<\beta$.

\footnotetext{
${ }^{5}$ From standard extension theorems of finite Borel measures as [7, Corollary 1.6.2] (whose proof is also valid for complex measures as the reader proves easily), there is only one regular complex Borel measure $\mu$ over $\mathbb{R}$ determined by the values $\mu([\alpha, \beta))$. If two PVMs $P, Q$ satisfy $\left\langle\phi \mid Q_{[\alpha, \beta)} \psi\right\rangle=\mu_{\phi, \psi}([\alpha, \beta))=$ $\left\langle\phi \mid P_{[\alpha, \beta)} \psi\right\rangle$ for all $\phi, \psi \in \mathrm{H}$ and $\alpha<\beta$ in $\mathbb{R}$, then it must also be $\left\langle\phi \mid Q_{E} \psi\right\rangle=\mu_{\phi, \psi}^{(Q)}(E)=\mu_{\phi, \psi}^{(P)}(E)=$ $\left\langle\phi \mid P_{E} \psi\right\rangle$ for every $E \in \mathscr{B}(\mathbb{R})$ and $\phi, \psi \in \mathrm{H}$, so that $P=Q$.
} 
Theorem 3.1 If $A$ and $B$ are as in Theorem 2.5 and $a, b \in \mathbb{R}$, the following identity holds for every $\alpha, \beta \in \mathbb{R}$ with $\alpha<\beta$,

$$
\begin{aligned}
P_{[\alpha, \beta)}^{(a A+b B)}= & s-\lim _{\epsilon \rightarrow 0^{+}} s-\lim _{N \rightarrow+\infty} \\
& \int_{\mathbb{R}^{2 N}} 1_{[\alpha, \beta)}^{(\epsilon)}\left(\frac{1}{N} \sum_{n=1}^{N}\left(a \lambda_{n}+b \mu_{n}\right)\right) d P_{\lambda_{1}}^{(A)} d P_{\mu_{1}}^{(B)} \cdots d P_{\lambda_{N}}^{(A)} d P_{\mu_{N}}^{(B)},
\end{aligned}
$$

where the $\epsilon$-parametrized class of $\mathscr{S}(\mathbb{R})$ functions

$$
1_{[\alpha, \beta)}^{(\epsilon)}(x):=\exp \left\{-\frac{\epsilon}{(x-\beta)^{2}}-\frac{\epsilon}{(x-\alpha)^{2}}\right\} 1_{(\alpha, \beta)}(x)+\exp \left\{-\frac{(x-\alpha)^{2}}{\epsilon}\right\}, \quad x \in \mathbb{R}
$$

pointwise converges to $1_{[\alpha, \beta)}$ for $\epsilon \rightarrow 0^{+}$.

Proof Observing that $\left\|1_{[\alpha, \beta)}^{(\epsilon)} \rightarrow 1_{[\alpha, \beta)}\right\|_{\infty} \leq 2$ and $1_{[\alpha, \beta)}^{(\epsilon)} \rightarrow 1_{[a, b)}$ pointwise for $\epsilon \rightarrow 0^{+}$, the Lebesgue dominated convergence theorem and the finiteness of the positive spectral measure $\mu_{\psi, \psi}^{(\overline{a A+b B})}(E):=\left\langle\psi \mid P_{E}^{(\overline{a A+b B})} \psi\right\rangle$ imply that

$$
\int_{\mathbb{R}}\left|1_{[\alpha, \beta)}^{(\epsilon)}(\tau)-1_{[\alpha, \beta)}(\tau)\right|^{2} \mathrm{~d} \mu_{\psi, \psi}^{(\overline{a A+b B})}(\tau) \stackrel{\epsilon \rightarrow 0^{+}}{\longrightarrow} 0 \text { for all } \psi \in \mathrm{H}
$$

Since $P_{[\alpha, \beta)}^{(\overline{a A+b B})}=1_{[\alpha, \beta)}(\overline{a A+b B})$ and on account of the general identity $\|f(\overline{a A+b B}) \psi\|^{2}=\int_{\mathbb{R}}|f(\tau)|^{2} \mathrm{~d} \mu_{\psi, \psi}^{(\overline{a A+b B})}(\tau)$, we have $P_{[\alpha, \beta)}^{(\overline{a A+b B})}=\mathrm{s}-\lim _{\epsilon \rightarrow 0^{+}} 1_{[\alpha, \beta)}^{(\epsilon)}$ $(\overline{a A+b B})$. Moreover, $1_{[\alpha, \beta)}^{(\epsilon)} \in \mathscr{S}(\mathbb{R})$, so that it belongs to $\mathscr{F}(\mathbb{R})$ and we can apply Theorem 2.5 to the right-hand side obtaining (38).

Remark 3.2 (1) A statement analogous to that in Theorem 3.1 can be proved for the elements $P_{(\gamma, \alpha]}^{(\overline{a A+b B})}$ with $\gamma<\alpha$. Knowledge of $P_{(\gamma, \alpha]}^{(\overline{a A+b B})}$ and $P_{[\alpha, \beta)}^{(\overline{a A+b B})}$ permits one to construct the atom $P_{\{\alpha\}}^{(\overline{a A+b B})}$ since $P_{(\gamma, \alpha]}^{(\overline{a A+b B})} P_{[\alpha, \beta)}^{(\overline{a A+b B})}=P_{(\gamma, \alpha] \cap[\alpha, \beta)}^{(\overline{a A+b B})}=$ $P_{\{\alpha\}}^{(\overline{a A+b B})}$. Alternatively, $P_{\{\alpha\}}^{(\overline{a A+b B})}$ directly arises from (38) when using $1_{\{\alpha\}}^{(\epsilon)}(x):=$ $\exp \left\{-(x-\alpha)^{2} / \epsilon\right\}$ in place of $1_{[\alpha, \beta)}^{(\epsilon)}(x)$.

(2) If $\alpha$ and $\beta$ admit corresponding neighborhoods without intersection with the difference $\sigma(a A+b B) \backslash[\alpha, \beta)$, the $\epsilon$ limit can be removed from the left-hand side of (38) if replacing $1_{\{\alpha\}}^{(\epsilon)}$ with a map $1_{[\alpha, \beta)}^{\prime} \in \mathcal{D}(\mathbb{R})$ which constantly attains the value 1 over $[\alpha, \beta)$ and rapidly vanishes on $\sigma(a A+b B) \backslash[\alpha, \beta)$.

(3) Theorem 3.1 proves that the PVM of $a A+b B$ can be determined from the PVMs of the operators $A$ and $B$. With a slightly different approach (assuming $a=b=1$ for the sake of simplicity), it is also possible to prove a more direct relation between the Fourier transform of the spectral measures $\mu_{\psi, \varphi}^{(\overline{A+B})}$ and those of the spectral measures 
$\mu_{\psi, \varphi}^{(A)}$ and $\mu_{\psi, \varphi}^{(B)}$. For that, let $\psi, \varphi \in \mathrm{H}$ and let $\left\{\psi_{k}\right\}_{k \in \mathbb{N}} \subset \mathrm{H}$ be a complete orthonormal basis of $\mathrm{H}$. From Trotter product formula, it follows that

$$
\begin{aligned}
\widehat{\mu}_{\psi, \varphi}^{(\overline{A+B})}(t) & =\left\langle\psi \mid \mathrm{e}^{i t(\overline{A+B})} \varphi\right\rangle \\
& =\lim _{N \rightarrow+\infty}\left\langle\psi \mid\left(\mathrm{e}^{\frac{i t}{N} A} \mathrm{e}^{\frac{i t}{N} B}\right)^{N} \varphi\right\rangle \\
& =\lim _{N \rightarrow+\infty} \sum_{k_{1}, \ldots, k_{N} \in \mathbb{N}}\left\langle\psi \mid \mathrm{e}^{\frac{i t}{N} A} \psi_{k_{1}}\right\rangle\left\langle\psi_{k_{1}} \mid \mathrm{e}^{\frac{i t}{N} B} \psi_{k_{2}}\right\rangle \cdots\left\langle\psi_{k_{N-1}} \mid \mathrm{e}^{\frac{i t}{N} A} \psi_{k_{N}}\right\rangle\left\langle\psi_{k_{N}} \mid \mathrm{e}^{\frac{i t}{N} B} \varphi\right\rangle \\
& =\lim _{N \rightarrow+\infty} \sum_{k_{1}, \ldots, k_{N} \in \mathbb{N}} \widehat{\mu}_{\psi, \psi_{k_{1}}}^{(A)}\left(\frac{i t}{N}\right) \widehat{\mu}_{\psi_{k_{1}}, \psi_{k_{2}}}^{(B)}\left(\frac{i t}{N}\right) \cdots \widehat{\mu}_{\psi_{k_{N-1}}, \psi_{k_{N}}}^{(A)}\left(\frac{i t}{N}\right) \widehat{\mu}_{\psi_{k_{N}}, \varphi}^{(B)}\left(\frac{i t}{N}\right) .
\end{aligned}
$$

Together with the injectivity of the Fourier transform for finite measure - c.f. Eq. (16)(40) provides another (more indirect) relation between $P^{(\overline{A+B})}$ and $P^{(A)}, P^{(B)}$. Notice that Eq. (40) can be proved without invoking either Lemma 2.2 or Theorem 2.5although it can be seen as an application of Theorem 2.5 for the case $f=1_{\mathbb{R}}=f_{\delta} \in$ $\mathscr{F}(\mathbb{R}), \delta$ denoting the Dirac measure centered at 0 .

Example 3.3 We provide here an explicit computation of formula (32) which is shown to match with the exact result-c.f. Theorem 2.5 and part (3) Remark 3.2. Let $\mathrm{H}=$ $L^{2}(\mathbb{R}, \mathrm{d} x)$ and let $A:=X$ be (with obvious domain) the self-adjoint position operator $(X \psi)(x):=x \psi(x)$, and $B:=P$ the self-adjoint momentum operator (the unique self-adjoint extension of $(B \psi)(x):=i \frac{\mathrm{d} \psi}{\mathrm{d} x}(x)$ for $\left.\psi \in \mathscr{S}(\mathbb{R})\right)^{6}$. It turns out that $\mathscr{S}(\mathbb{R}) \subset D(A) \cap D(B)$ and $a A+b B$ is essentially self-adjoint thereon for every $a, b \in \mathbb{R}$, its closure being a generator of the Weyl algebra [25] according to Stone's theorem. As a consequence, the previously developed theory can be applied to the pair $A, B$.

Taking advantage of Weyl algebra commutation relations, namely

$$
\mathrm{e}^{i a X} \mathrm{e}^{i b P}=\mathrm{e}^{-\frac{i}{2} a b} \mathrm{e}^{i a X+i b P}, \quad a, b \in \mathbb{R},
$$

we have that for all $\phi, \psi \in \mathrm{H}$

$$
\widehat{\mu}_{\phi, \psi}^{(\overline{A+B})}(t)=\left\langle\phi \mid \mathrm{e}^{i t(\overline{A+B})} \psi\right\rangle=\mathrm{e}^{-\frac{i}{2} t^{2}}\left\langle\phi \mid \mathrm{e}^{i t X} \mathrm{e}^{i t P} \psi\right\rangle=\mathrm{e}^{-\frac{i}{2} t^{2}} \int_{\mathbb{R}} \overline{\phi\left(x_{1}\right)} \mathrm{e}^{i t x_{1}} \psi\left(x_{1}-t\right) \mathrm{d} x_{1} .
$$

We argue that equation (32) reproduces the exact result (41). A simple computation shows that, if $E \in \mathscr{B}(\mathbb{R})$,

$$
\left(P_{E}^{(A)} \psi\right)(x)=1_{E}(x) \psi(x), \quad \widehat{\left(P_{E}^{(B)} \psi\right)}(k)=1_{E}(k) \widehat{\psi}(k),
$$

\footnotetext{
${ }^{6}$ We recall that our convention for the Fourier transform is $\widehat{g}(k):=(2 \pi)^{-1 / 2} \int_{\mathbb{R}} g(x) \mathrm{e}^{i x k} \mathrm{~d} x, g(x)=$ $(2 \pi)^{-1 / 2} \int_{\mathbb{R}} \widehat{g}(k) \mathrm{e}^{-i k x} d k$ for $g \in \mathscr{S}(\mathbb{R})$ —.f. Eq. (15).
} 


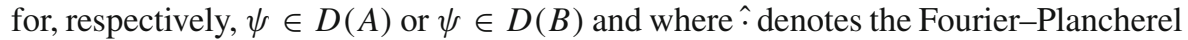
transform. With this information, we wish to compute the limit as $N \rightarrow+\infty$ of

$$
\begin{aligned}
& \left\langle\phi \mid \int_{\mathbb{R}^{2 N}} \mathrm{e}^{i t \sum_{\ell=1}^{N}\left(x_{\ell}+k_{\ell}\right)} \mathrm{d} P_{x_{1}}^{(A)} \mathrm{d} P_{k_{1}}^{(B)} \cdots \mathrm{d} P_{x_{N}}^{(A)} \mathrm{d} P_{k_{N}}^{(B)} \psi\right\rangle \\
& =\int_{\mathbb{R}^{2 N}} \mathrm{e}^{\frac{i t}{N} \sum_{\ell=1}^{N}\left(x_{\ell}+k_{\ell}\right)} \rho_{\phi, \psi}\left(x_{1}, \ldots, k_{N}\right) \mathrm{d} x_{1} \mathrm{~d} k_{1} \cdots \mathrm{d} x_{N} \mathrm{~d} k_{N},
\end{aligned}
$$

where $\rho_{\phi, \psi}\left(x_{1}, \ldots, k_{N}\right)=\frac{1}{(2 \pi)^{N-\frac{1}{2}}} \overline{\phi\left(x_{1}\right)} \prod_{\ell=1}^{N-1} \mathrm{e}^{i k_{\ell}\left(x_{\ell+1}-x_{\ell}\right)} \mathrm{e}^{-i k_{N} x_{N}} \widehat{\psi}\left(k_{N}\right)$. Equality (43) follows from (25) and (42) by applying Fourier-Plancherel transform and its inverse $N$ times. Notice that we can avoid the regularization $Q_{n}$ by considering $\rho$ as a distribution-there is no integration in $t$. A direct computation leads to

$$
\begin{aligned}
& \int_{\mathbb{R}^{2 N}} \mathrm{e}^{\frac{i t}{N} \sum_{\ell=1}^{N}\left(x_{\ell}+k_{\ell}\right)} \rho_{\phi, \psi}\left(x_{1}, \ldots, k_{N}\right) \mathrm{d} x_{1} \cdots \mathrm{d} x_{N} d k_{1} \cdots \mathrm{d} k_{N} \\
&=\int_{\mathbb{R}^{2 N}} \frac{\mathrm{e}^{\frac{i t}{N} \sum_{\ell=1}^{N} x_{\ell}} \overline{\phi\left(x_{1}\right)}}{(2 \pi)^{N-1 / 2}} \prod_{\ell=1}^{N-1} \mathrm{e}^{i k_{\ell}\left(x_{\ell+1}-x_{\ell}+\frac{t}{N}\right)} \mathrm{e}^{-i k_{N}\left(x_{N}-\frac{t}{N}\right)} \widehat{\psi}\left(k_{N}\right) \mathrm{d} x_{1} \\
& \cdots \mathrm{d} x_{N} d k_{1} \cdots \mathrm{d} k_{N} \\
&= \int_{\mathbb{R}^{N}} \mathrm{e}^{\frac{i t}{N} \sum_{\ell=1}^{N} x_{\ell} \overline{\phi\left(x_{1}\right)}} \prod_{\ell=1}^{N-1} \delta\left(x_{\ell+1}-x_{\ell}+\frac{t}{N}\right) \psi\left(x_{N}-\frac{t}{N}\right) \mathrm{d} x_{1} \cdots \mathrm{d} x_{N} \\
&= \int_{\mathbb{R}} \mathrm{e}^{i t x_{1}-\frac{i}{2} t^{2} \frac{N(N-1)}{N^{2}}} \overline{\phi\left(x_{1}\right)} \psi\left(x_{1}-t\right) \mathrm{d} x_{1} \\
& \stackrel{N \rightarrow+\infty}{\longrightarrow} \mathrm{e}^{-\frac{i}{2} t^{2}} \int_{\mathbb{R}} \mathrm{e}^{i t x_{1}} \overline{\phi\left(x_{1}\right)} \psi\left(x_{1}-t\right) \mathrm{d} x_{1},
\end{aligned}
$$

where in the Dirac delta distributions we are ensured that $x_{\ell}=x_{1}-\frac{\ell}{N} t$ for $\ell=$ $2, \ldots, N$.

\subsection{Relation with Feynman integration}

The next application proves the close relation between formula (32) and the notion of Feynman integral in phase space. Let us consider now a slight modification of Example 3.3. Let $\mathrm{H}:=L^{2}(\mathbb{R}, \mathrm{d} x)$ and let $(A \psi)(x):=x^{2} \psi(x)$ and $B:=P^{2}$ be, respectively, the square of the self-adjoint position and momentum operators defined in Example 3.3. Further let $a=\Omega^{2} / 2$ and $b=(2 m)^{-1}$, with $m, \Omega>0$ constants. It is well known that $A+B$ is essentially self-adjoint over $\mathscr{S}(\mathbb{R}) \subset D(A) \cap D(B)$ and $\overline{A+B}$ coincides with the harmonic oscillator Hamiltonian operator $H$

$$
H=\overline{\frac{P^{2}}{2 m}+\frac{\Omega^{2}}{2} X^{2}} .
$$

Let us recall that if $T$ is a self-adjoint operator on $\mathrm{H}$ with spectral measure $P^{(T)}$ and $h: \mathbb{R} \rightarrow \mathbb{R}$ is a Borel measurable map, the spectral measure $P^{(h(T))}$ of the self- 
adjoint operator $h(T)$ (defined via functional calculus out of $T$ ) is related to $P^{(T)}$ by the formula

$$
\int_{\mathbb{R}} f(\lambda) \mathrm{d} P_{\lambda}^{(h(T))}=\int_{\mathbb{R}} f(h(\lambda)) \mathrm{d} P_{\lambda}^{(T)}
$$

for any Borel-measurable function $f: \mathbb{R} \rightarrow \mathbb{C}$.

As a consequence, if $f \in \mathscr{F}(\mathbb{R})$ and using $h: \mathbb{R} \ni r \mapsto r^{2},(32)$ implies

$$
f(H)=s-\lim _{N \rightarrow \infty} \int_{\mathbb{R}^{2 N}} f\left(\frac{1}{N} \sum_{n=1}^{N}\left(\frac{k_{n}^{2}}{2 m}+\frac{\Omega^{2}}{2} x_{n}^{2}\right)\right) \mathrm{d} P_{x_{1}}^{(X)} \mathrm{d} P_{k_{1}}^{(P)} \cdots \mathrm{d} P_{x_{N}}^{(X)} \mathrm{d} P_{k_{N}}^{(P)} .
$$

If we now choose the function $f \in \mathscr{F}(\mathbb{R})$ of the form $f(\lambda)=\mathrm{e}^{-i t \lambda}$, with $t \in \mathbb{R}$, then for any $\phi, \psi \in \mathrm{H}$ we have the following representation for the matrix elements of the unitary evolution operator $U(t)=\mathrm{e}^{-i t H}$

$$
\left\langle\phi \mid \mathrm{e}^{-i t H} \psi\right\rangle=\lim _{N \rightarrow \infty} \int_{\mathbb{R}^{2 N}} \mathrm{e}^{-i \sum_{n=1}^{N}\left(\frac{k_{n}^{2}}{2 m}+\frac{\Omega^{2}}{2} x_{n}^{2}\right) \frac{t}{N}}\left\langle\phi \mid \mathrm{d} P_{x_{1}}^{(X)} \mathrm{d} P_{k_{1}}^{(P)} \cdots \mathrm{d} P_{x_{N}}^{(X)} \mathrm{d} P_{k_{N}}^{(P)} \psi\right\rangle .
$$

Analogously to the derivation of equality (43), iterated applications of Fourier transform and its inverse yield

$$
\left\langle\phi \mid \mathrm{e}^{-i t H} \psi\right\rangle=\lim _{\substack{N \rightarrow \infty \\ \mathrm{d} x_{1} \cdots \mathrm{d} x_{N+1} \mathrm{~d} k_{1} \cdots \mathrm{d} k_{N}}} \int_{\mathbb{R}^{2 N+1}} \frac{\mathrm{e}^{-i \sum_{n=1}^{N}\left(\frac{k_{n}^{2}}{2 m}+\frac{\Omega^{2}}{2} x_{n}^{2}\right) \frac{t}{N}}}{(2 \pi)^{N}} \overline{\phi\left(x_{1}\right)} \psi\left(x_{N+1}\right) \mathrm{e}^{i \sum_{\ell=1}^{N} k_{\ell}\left(x_{\ell+1}-x_{\ell}\right)}
$$

where we recall that the integrals over $\mathbb{R}^{2 N}$ are just a formal symbol standing for the limit of the regularizing procedure described in (25), since in this case the distribution (27) is not associated with a complex measure on $\mathscr{B}\left(\mathbb{R}^{2 N}\right)$. Formula (46) admits an interpretation in terms of a phase space Feynman path integral representation for the time evolution operator $U(t)$, as discussed, e.g., in [1,17]. Indeed, it is possible to look at the exponent appearing in the integral on the right-hand side of (46), namely the function

$$
S\left(x_{1}, \ldots, x_{N+1}, k_{1}, \ldots, k_{N}\right)=\sum_{l=1}^{N} k_{l}\left(x_{l+1}-x_{l}\right)-\sum_{n=1}^{N}\left(\frac{k_{n}^{2}}{2 m}+\frac{\Omega^{2}}{2} x_{n}^{2}\right) \frac{t}{N}
$$

as the Riemann sum approximation of the classical action functional in the Hamiltonian formulation:

$$
S[q, p]=\int_{0}^{t} p(s) \mathrm{d} q(s)-\int_{0}^{t} h(q(s), p(s)) \mathrm{d} s,
$$


where $h: \mathbb{R}^{2} \rightarrow \mathbb{R}$ is the classical Hamiltonian of the harmonic oscillator, i.e.,

$$
h(x, k)=\frac{k^{2}}{2 m}+\frac{\Omega^{2}}{2} x^{2}, \quad(x, k) \in \mathbb{R}^{2} .
$$

A completely similar discussion can be repeated in the case the harmonic oscillator potential is replaced with a more general potential, namely a measurable map $V: \mathbb{R} \rightarrow$ $\mathbb{R}$ such that the sum of the associated multiplication operator $M_{V}: D\left(M_{V}\right) \subset \mathrm{H} \rightarrow \mathrm{H}$ and $B=P^{2}$ is essentially self-adjoint. We stress that the limit as $N \rightarrow \infty$ on the right-hand side of (46) cannot be interpreted in terms of a well-defined integral over an infinite-dimensional space of paths $(q, p):[0, t] \rightarrow \mathbb{R}^{2}$, formally written (in Feynman path integral notation) as

$$
\left\langle\phi \mid \mathrm{e}^{-i t H} \psi\right\rangle=\int_{\left\{(q, p):[0, t] \rightarrow \mathbb{R}^{2}\right\}} \mathrm{e}^{i S[q, p]} \bar{\phi}(q(t)) \psi(q(0)) \mathrm{d} q \mathrm{~d} p
$$

since it is impossible to construct a corresponding complex measure, as extensively discussed in [2] and in Sect. 4.2.

\section{3 (Semi)bounded $A, B$, Jordan product, polynomials, Feynman-Kac formula}

If the self-adjoint operators $A$ and $B$ are bounded, then $a A+b B \in \mathfrak{B}(\mathrm{H})$ is defined for all $a, b \in \mathbb{R}$ and (22) permits us to extend (32) to the case of $f \in C^{\infty}(\mathbb{R})$. A similar result is valid when $A$ and $B$ are bounded from below.

Let us start with $A, B \in \mathfrak{B}(\mathrm{H})$. For any choice of $a, b \in \mathbb{R}$, we then have

$$
\begin{aligned}
\int_{\mathbb{R}^{2 N}} f\left(\frac { 1 } { N } \sum _ { l = 1 } ^ { N } \left(a \lambda_{l}\right.\right. & \left.\left.+b \mu_{l}\right)\right) \mathrm{d} \nu_{\phi, \psi, Q}^{(N, n)}\left(\lambda_{1}, \mu_{1}, \ldots, \lambda_{N}, \mu_{N}\right) \\
& =\int_{\mathbb{R}^{2 N}} \tilde{f}\left(\frac{1}{N} \sum_{l=1}^{N}\left(a \lambda_{l}+b \mu_{l}\right)\right) \mathrm{d} \nu_{\phi, \psi, Q}^{(N, n)}\left(\lambda_{1}, \mu_{1}, \ldots, \lambda_{N}, \mu_{N}\right)
\end{aligned}
$$

for every $n \in \mathbb{N}$, if $\tilde{f} \in \mathscr{S}(\mathbb{R})$ is such that $\tilde{f}(x)=f(x)$ if $x \in[-\|a A\|-$ $\|b B\|,\|a A\|+\|b\|]$. As a consequence, (23) is still valid with $\tilde{f}$ in place of $f$ on the right-hand side, and the limit does not depend neither on the choice of the regularizing sequence $Q:=\left\{Q_{n}\right\}_{n \in \mathbb{N}}$ nor on the choice of $\tilde{f}$ :

$$
\begin{aligned}
& \lim _{n \rightarrow \infty} \int_{\mathbb{R}^{2 N}} f\left(\frac{1}{N} \sum_{l=1}^{N}\left(a \lambda_{l}+b \mu_{l}\right)\right) \mathrm{d} \nu_{\phi, \psi, Q}^{(N, n)}\left(\lambda_{1}, \mu_{1}, \ldots, \lambda_{N}, \mu_{N}\right) \\
& =\int_{\mathbb{R}}\left\langle\phi \mid\left(\mathrm{e}^{i \frac{t}{N} a A} \mathrm{e}^{i \frac{t}{N} b B}\right)^{N} \psi\right\rangle \mathrm{d} \nu_{\tilde{f}}(t) .
\end{aligned}
$$


A similar result is found when $A$ and $B$ are (unbounded) self-adjoint operators which are bounded from below and $a, b \geq 0$ are such that $a A+b B$ is essentially selfadjoint. In this case $\overline{a A+b B}$ is bounded from below as well with inf $\sigma(\overline{a A+b B}) \geq$ $a \inf \sigma(A)+b \inf \sigma(B)$. With the said $A, B, a, b$, both (47) and (48) are true provided $f \in C^{\infty}(\mathbb{R}), \tilde{f} \in \mathscr{S}(\mathbb{R})$, and $f(x)=\tilde{f}(x)$ for $x \geq a \inf \sigma(A)+b \inf \sigma(B)$.

Identity (48) is sufficient in both cases for giving the following definition based on Riesz' lemma.

Definition 3.4 Assume that one of the following two cases holds

(a) $A, B \in \mathfrak{B}(\mathrm{H})$ are self-adjoint, $a, b \in \mathbb{R}$, and $f \in C^{\infty}(\mathbb{R})$;

(b) $A, B$ are self-adjoint and bounded from below, $a, b \geq 0, a A+b B$ is essentially self-adjoint, and $f \in C^{\infty}(\mathbb{R})$ is such that there exists $\tilde{f} \in \mathscr{S}(\mathbb{R})$ for which $f(x)=\tilde{f}(x)$ for $x \geq a \inf \sigma(A)+b \inf \sigma(B)$.

Then,

$$
\int_{\mathbb{R}^{2 N}} f\left(\frac{1}{N} \sum_{n=1}^{N}\left(a \lambda_{n}+b \mu_{n}\right)\right) \mathrm{d} P_{\lambda_{1}}^{(A)} \mathrm{d} P_{\mu_{1}}^{(B)} \cdots \mathrm{d} P_{\lambda_{N}}^{(A)} \mathrm{d} P_{\mu_{N}}^{(B)} \in \mathfrak{B}(\mathrm{H})
$$

is defined as the unique operator satisfying (25).

This definition is an evident extension of the definition for $f \in \mathscr{F}(\mathbb{R})$ appearing in Theorem 2.3 because, if $\tilde{f} \in \mathscr{S}(\mathbb{R}) \subset \mathscr{F}(\mathbb{R})$ is defined as above with respect to $f$, then

$$
\begin{aligned}
\int_{\mathbb{R}^{2 N}} f\left(\frac{1}{N} \sum_{n=1}^{N}\left(a \lambda_{n}+b \mu_{n}\right)\right) \mathrm{d} P_{\lambda_{1}}^{(A)} \mathrm{d} P_{\mu_{1}}^{(B)} \cdots \mathrm{d} P_{\lambda_{N}}^{(A)} \mathrm{d} P_{\mu_{N}}^{(B)} \\
=\int_{\mathbb{R}^{2 N}} \tilde{f}\left(\frac{1}{N} \sum_{n=1}^{N}\left(a \lambda_{n}+b \mu_{n}\right)\right) \mathrm{d} P_{\lambda_{1}}^{(A)} \mathrm{d} P_{\mu_{1}}^{(B)} \cdots \mathrm{d} P_{\lambda_{N}}^{(A)} \mathrm{d} P_{\mu_{N}}^{(B)} .
\end{aligned}
$$

The remaining properties (i)-(iv) of Theorem 2.3 hold as well provided $\|f\|_{\mathscr{F}}$ is replaced for $\|\tilde{f}\|_{\mathscr{F}}$ on the right-hand side of (26) in (ii).

With this extended definition, Theorem 2.5 is still valid as the reader immediately proves noticing in particular that from (3) and (4), we have

$$
f(a A+b B)=\tilde{f}(a A+b B) .
$$

Proposition 3.5 If either (a) or (b) in Definition 3.4 is valid, then

$$
\begin{gathered}
f(\overline{a A+b B})=s-\lim _{N \rightarrow+\infty} \int_{\mathbb{R}^{2 N}} f\left(\frac{1}{N} \sum_{n=1}^{N}\left(a \lambda_{n}+b \mu_{n}\right)\right) \mathrm{d} P_{\lambda_{1}}^{(A)} \mathrm{d} P_{\mu_{1}}^{(B)} \\
\cdots \mathrm{d} P_{\lambda_{N}}^{(A)} \mathrm{d} P_{\mu_{N}}^{(B)}
\end{gathered}
$$


If with $A, B \in \mathfrak{B}(\mathrm{H})$, Definition 3.4 applies in particular to the operator $a A+b B$ as in (51) below, when referring to the smooth map $f: \mathbb{R} \ni x \mapsto x \in \mathbb{R}$. That identity shows how the real linear space structure of the algebra of bounded observables can be constructed employing the PVMs of the involved observables only. The whole algebra of bounded observables $\mathfrak{B}(\mathrm{H})$ has another natural operation that is the (nonassociative) Jordan product:

$$
A \circ B:=\frac{1}{2}(A B+B A), \quad \text { for } A=A^{*}, B=B^{*}, A, B \in \mathfrak{B}(\mathrm{H}) \text {. }
$$

The Jordan product of $A$ and $B$ admits an expansion similar to (51) where, again, only the PVMs of $A$ and $B$ is used. These results can be further generalized as stated in the last item of the following theorem which concerns polynomials.

Theorem 3.6 Let $A, B \in \mathfrak{B}(H)$ be self-adjoint operators, with $H$ separable, whose $P V M s$ are, respectively, denoted by $P^{(A)}$ and $P^{(B)}$. Let $v_{\phi, \psi, Q}^{(N, n)}: \mathscr{B}\left(\mathbb{R}^{2 N}\right) \rightarrow \mathbb{C}$ be the complex measures defined as in (21). The following facts are true.

(a) If $a, b \in \mathbb{R}$,

$$
a A+b B=s-\lim _{N \rightarrow+\infty} \int_{\mathbb{R}^{2 N}}\left(a \sum_{n=1}^{N} \frac{\lambda_{n}}{N}+b \sum_{n=1}^{N} \frac{\mu_{n}}{N}\right) \mathrm{d} P_{\lambda_{1}}^{(A)} \mathrm{d} P_{\mu_{1}}^{(B)} \cdots \mathrm{d} P_{\lambda_{N}}^{(A)} \mathrm{d} P_{\mu_{N}}^{(B)}
$$

(b) The Jordan product of $A$ and $B$ satisfies

$$
A \circ B=s-\lim _{N \rightarrow+\infty} \int_{\mathbb{R}^{2 N}}\left(\sum_{n=1}^{N} \frac{\lambda_{n}}{N}\right)\left(\sum_{n=1}^{N} \frac{\mu_{n}}{N}\right) \mathrm{d} P_{\lambda_{1}}^{(A)} \mathrm{d} P_{\mu_{1}}^{(B)} \cdots \mathrm{d} P_{\lambda_{N}}^{(A)} \mathrm{d} P_{\mu_{N}}^{(B)}
$$

where the operator after the symbol of limit is the unique operator such that

$$
\begin{aligned}
& \left\langle\phi \mid \int_{\mathbb{R}^{2 N}}\left(\sum_{n=l}^{N} \frac{\lambda_{l}}{N}\right)\left(\sum_{n=l}^{N} \frac{\mu_{l}}{N}\right) \mathrm{d} P_{\lambda_{1}}^{(A)} \mathrm{d} P_{\mu_{1}}^{(B)} \cdots \mathrm{d} P_{\lambda_{N}}^{(A)} \mathrm{d} P_{\mu_{N}}^{(B)} \psi\right\rangle \\
& =\lim _{n \rightarrow \infty} \int_{\mathbb{R}^{2 N}}\left(\sum_{l=1}^{N} \frac{\lambda_{l}}{N}\right)\left(\sum_{l=1}^{N} \frac{\mu_{l}}{N}\right) \mathrm{d} v_{\phi, \psi, Q}^{(N, n)}\left(\lambda_{1}, \mu_{1}, \ldots, \lambda_{N}, \mu_{N}\right)
\end{aligned}
$$

for all $\phi, \psi \in H$.

(c) Assume $p, q=0,1,2, \ldots$ and let $S_{p+q}\left(A^{p} B^{q}\right)$ denote the self-adjoint operator consisting of the symmetrized product of $p$ copies of $A$ and $q$ copies of $B$,

$$
S_{p+q}\left(A^{p} B^{q}\right):=\left(\begin{array}{c}
p+q \\
q
\end{array}\right)^{-1}\left(A^{p} B^{q}+A^{p-1} B A B^{q-1}+\cdots+B^{q} A^{p}\right) .
$$


If only a finite number of coefficients $c_{p, q} \in \mathbb{R}$ do not vanish, then

$$
\begin{aligned}
& \sum_{p, q} c_{p, q} S_{p+q}\left(A^{p} B^{q}\right) \\
& \quad=s^{-} \lim _{N \rightarrow+\infty} \int_{\mathbb{R}^{2 N}} \sum_{p, q} c_{p, q}\left(\sum_{n=1}^{N} \frac{\lambda_{n}}{N}\right)^{p}\left(\sum_{n=1}^{N} \frac{\mu_{n}}{N}\right)^{q} \mathrm{~d} P_{\lambda_{1}}^{(A)} \mathrm{d} P_{\mu_{1}}^{(B)} \cdots \mathrm{d} P_{\lambda_{N}}^{(A)} \mathrm{d} P_{\mu_{N}}^{(B)}
\end{aligned}
$$

where the operator after the symbol of limit is the unique operator such that

$$
\begin{aligned}
& \left\langle\phi \mid \int_{\mathbb{R}^{2 N}} \sum_{p, q} c_{p, q}\left(\sum_{l=1}^{N} \frac{\lambda_{l}}{N}\right)^{p}\left(\sum_{l=1}^{N} \frac{\mu_{l}}{N}\right)^{q} \mathrm{~d} P_{\lambda_{1}}^{(A)} \mathrm{d} P_{\mu_{1}}^{(B)} \cdots \mathrm{d} P_{\lambda_{N}}^{(A)} \mathrm{d} P_{\mu_{N}}^{(B)} \psi\right\rangle \\
& =\lim _{n \rightarrow \infty} \int_{\mathbb{R}^{2 N}} \sum_{p, q} c_{p, q}\left(\sum_{l=1}^{N} \frac{\lambda_{l}}{N}\right)^{p}\left(\sum_{l=1}^{N} \frac{\mu_{l}}{N}\right)^{q} \mathrm{~d} v_{\phi, \psi, Q}^{(N, n)}\left(\lambda_{1}, \mu_{1}, \ldots, \lambda_{N}, \mu_{N}\right),
\end{aligned}
$$

for all $\phi, \psi \in H$.

The limit in the right-hand side of (53) and (55) does not depend on the choice of the regularizing sequence $Q$.

Proof We only have to prove (b) and (c). Regarding (b), it essentially relies on the elementary decomposition $x y=\frac{1}{2}(x+y)^{2}-\frac{1}{2} x^{2}-\frac{1}{2} y^{2}$, which implies

$$
\begin{gathered}
2 \int_{\mathbb{R}^{2 N}}\left(\sum_{l=1}^{N} \frac{\lambda_{l}}{N}\right)\left(\sum_{l=1}^{N} \frac{\mu_{l}}{N}\right) \mathrm{d} v_{\phi, \psi, Q}^{(N, n)}\left(\lambda_{1}, \mu_{1}, \ldots, \lambda_{N}, \mu_{N}\right) \\
=\int_{\mathbb{R}^{2 N}}\left(\frac{1}{N} \sum_{l=1}^{N}\left(\lambda_{l}+\mu_{l}\right)\right)^{2} \mathrm{~d} \nu_{\phi, \psi, Q}^{(N, n)}\left(\lambda_{1}, \mu_{1}, \ldots, \lambda_{N}, \mu_{N}\right) \\
\quad-\int_{\mathbb{R}^{2 N}}\left(\frac{1}{N} \sum_{l=1}^{N} \lambda_{l}\right)^{2} \mathrm{~d} v_{\phi, \psi, Q}^{(N, n)}\left(\lambda_{1}, \mu_{1}, \ldots, \lambda_{N}, \mu_{N}\right) \\
-\int_{\mathbb{R}^{2 N}}\left(\frac{1}{N} \sum_{l=1}^{N} \mu_{l}\right)^{2} \mathrm{~d} v_{\phi, \psi, Q}^{(N, n)}\left(\lambda_{1}, \mu_{1}, \ldots, \lambda_{N}, \mu_{N}\right) .
\end{gathered}
$$

As discussed above, the limits for $n \rightarrow+\infty$ of the final three addends exist and do not depend on the used sequence $Q$. We conclude that the right-hand side of (53) exists and does not depend on $Q$. More precisely, from (48), 


$$
\begin{aligned}
& \lim _{n \rightarrow+\infty} \int_{\mathbb{R}^{2 N}}\left(\sum_{l=1}^{N} \frac{\lambda_{l}}{N}\right)\left(\sum_{l=1}^{N} \frac{\mu_{l}}{N}\right) \mathrm{d} v_{\phi, \psi, Q}^{(N, n)}\left(\lambda_{1}, \mu_{1}, \ldots, \lambda_{N}, \mu_{N}\right) \\
& =\frac{1}{2} \int_{\mathbb{R}}\left\langle\phi \mid\left(\mathrm{e}^{i \frac{t}{N} A} \mathrm{e}^{i \frac{t}{N} B}\right)^{N} \psi\right\rangle \mathrm{d} v_{\tilde{f}}(t)-\frac{1}{2} \int_{\mathbb{R}}\left\langle\phi \mid \mathrm{e}^{i t A} \psi\right\rangle \mathrm{d} v_{\tilde{f}}(t)-\frac{1}{2} \int_{\mathbb{R}}\left\langle\phi \mid \mathrm{e}^{i t B} \psi\right\rangle \mathrm{d} v_{\tilde{f}}(t)
\end{aligned}
$$

where $\tilde{f}: \mathbb{R} \rightarrow \mathbb{R}$ is such that $\tilde{f} \in \mathscr{S}(\mathbb{R})$ such that $\tilde{f}(x)=x^{2}$ for the values $x \in[-\|A\|-\|B\|,\|A\|+\|B\|]$. At this point, Riesz' lemma proves that there is a unique operator satisfying (53). Decomposing

$$
\left(\sum_{l=1}^{N} \frac{\lambda_{l}}{N}\right)\left(\sum_{l=1}^{N} \frac{\mu_{l}}{N}\right)=\frac{1}{2}\left(\frac{1}{N} \sum_{l=1}^{N}\left(\lambda_{\ell}+\mu_{\ell}\right)\right)^{2}-\frac{1}{2}\left(\frac{1}{N} \sum_{l=1}^{N} \lambda_{\ell}\right)^{2}-\frac{1}{2}\left(\frac{1}{N} \sum_{l=1}^{N} \mu_{\ell}\right)^{2}
$$

on the right-hand side of (52), and noticing that analogously to $x y=\frac{1}{2}(x+y)^{2}-$ $\frac{1}{2} x^{2}-\frac{1}{2} y^{2}$,

$$
A \circ B=\frac{1}{2}(A+B)^{2}-\frac{1}{2} A^{2}-\frac{1}{2} B^{2}
$$

(52) is an immediate consequence of (50) applied to the three addends on the right-hand sides of the two identities above. The proof of (c) is a straightforward generalization the proof of (b). It relies on the well-known Sylvester's result (see, e.g., the review part of [32]), that every real (or complex) homogeneous polynomial in $x$ and $y$ of degree $m$, in particular the monomial $x^{p} y^{q}$ (with $m:=p+q$ ), can be decomposed as $x^{p} y^{q}=\sum_{k}\left(a_{k} x+b_{k} y\right)^{m}$ for a finite number $(\leq m+1)$ of constants $a_{k}, b_{k} \in \mathbb{R}$ (resp. $\mathbb{C})$, and the fact that this result easily implies $S_{p+q}\left(A^{p} B^{q}\right)=\sum_{k}\left(a_{k} A+b_{k} B\right)^{m}$.

Remark 3.7 Item (c) proves the existence of a linear map associating each real polynomial $p$ on $\mathbb{R}^{2}$ to a suitably symmetrized (as in the left-hand side of (54)) polynomial of the operators $A$ and $B$. One naturally wonders if that map is a quantization map [18]. It is not possible to answer the question without further information on the *-algebra generated by $A$ and $B$. As a matter of fact, a necessary condition is that the aforementioned map transforms a Poisson structure on the space of polynomials to a real Lie-algebra structure on the space of the said polynomials of $A$ and $B$, whose Lie bracket is the commutator of operators (more precisely $i[\cdot, \cdot]$ ). In the general case, the space of those polynomials of operators is not a Lie algebra, unless $A$ and $B$ satisfy some further relations, in particular, it must be possible to expand $i[A, B]$ as a real polynomial of $A$ and $B$ which is symmetrized as said.

Case (b) in Proposition 3.5 has an interesting application which proves the interplay of formula (32) and the so-called Feynman-Kac formula.

Example 3.8 Let us consider again the setting of Sect. 3.2. Since in this case both operators $A$ and $B$ have positive spectrum, we can apply formula (50) also for $f$ with exponential growth. In particular, we can choose $f: \mathbb{R} \rightarrow \mathbb{R}$ of the form $f(\lambda)=\mathrm{e}^{-\tau \lambda}$, 
with $\tau>0$, obtaining the following representation for the matrix elements of the semigroup generated by the self-adjoint operator $H$ [see Eq. (44)]:

$$
\begin{gathered}
\left\langle\phi \mid \mathrm{e}^{-\tau H} \psi\right\rangle=\lim _{N \rightarrow \infty} \frac{1}{(2 \pi)^{N}} \int_{\mathbb{R}^{2 N+1}} \mathrm{e}^{-\sum_{n=1}^{N}\left(\frac{k_{n}^{2}}{2 m}+\frac{\Omega^{2}}{2} x_{n}^{2}\right) \frac{\tau}{N}} \overline{\phi\left(x_{1}\right)} \psi\left(x_{N+1}\right) \mathrm{e}^{i \sum_{\ell=1}^{N} k_{\ell}\left(x_{\ell+1}-x_{\ell}\right)} \\
\mathrm{d} x_{1} \cdots \mathrm{d} x_{N+1} \mathrm{~d} k_{1} \cdots \mathrm{d} k_{N}
\end{gathered}
$$

If $\psi \in L^{1}(\mathbb{R}, \mathrm{d} x)$, the integrals on $\mathbb{R}^{2 N+1}$ appearing on the right-hand side are absolutely convergent thanks to the fast decaying properties of the exponentials appearing in the integrand. In particular, in this case, by applying Fubini theorem and integrating with respect to $k_{1}, \ldots, k_{N}$, we get

$$
\begin{aligned}
\left\langle\phi \mid \mathrm{e}^{-\tau H} \psi\right\rangle= & \lim _{N \rightarrow \infty}\left(\frac{m N}{2 \pi \tau}\right)^{N / 2} \\
& \int_{\mathbb{R}^{N+1}} \mathrm{e}^{-\sum_{n=1}^{N} \frac{\Omega^{2}}{2} x_{n}^{2} \frac{\tau}{N} \overline{\phi\left(x_{1}\right)} \psi\left(x_{N+1}\right) \mathrm{e}^{-\frac{m}{2} \sum_{\ell=1}^{N} \frac{\left(x_{\ell+1}-x_{\ell}\right)^{2}}{\tau / N}} \mathrm{~d} x_{1} \cdots \mathrm{d} x_{N+1} .}
\end{aligned}
$$

In this case, thanks to the presence of the Gaussian density

$$
\rho_{G}\left(x_{1}, \ldots, x_{N}\right)=\left(\frac{m N}{2 \pi \tau}\right)^{N / 2} \mathrm{e}^{-\frac{m}{2} \sum_{l=1}^{N} \frac{\left(x_{l}-x_{l-1}\right)^{2}}{\tau / N}}
$$

in the integrand, the limit for $N \rightarrow \infty$ of the finite-dimensional integrals appearing in the right-hand side of (59) can be interpreted in terms of an integral over the Banach space $C_{\tau}(\mathbb{R})$ of continuous paths $\omega:[0, \tau] \rightarrow \mathbb{R}$ endowed with the uniform norm, the corresponding Borel $\sigma$-algebra and the Wiener Gaussian measure $W$. By a standard argument (see, e.g., [38]), formula (59) yields the celebrated Feynman-Kac formula:

$$
\left\langle\phi \mid \mathrm{e}^{-\tau H} \psi\right\rangle=\int_{\mathbb{R}} \overline{\phi(x)} \int_{C_{\tau}(\mathbb{R})} \psi(x+\omega(\tau)) \mathrm{e}^{-\frac{\Omega^{2}}{2} \int_{0}^{\tau}(\omega(s)+x)^{2} \mathrm{~d} s} \mathrm{~d} W(\omega) \mathrm{d} x .
$$

As remarked at the end of Sect. 3.2, the discussion above generalizes to the case the harmonic oscillator potential is replaced with a Borel measurable non-negative map $V: \mathbb{R} \rightarrow \mathbb{R}$ such that the sum of the associated multiplication operator $M_{V}$ : $D\left(M_{V}\right) \subset \mathrm{H} \rightarrow \mathrm{H}$ and $B=P^{2}$ is essentially self-adjoint. Related ideas can be also found in [15,16,29] as well as in Lapidus' papers from the late 1980s [19-21].

\section{Counterexamples}

In this section, we provide a series of counterexamples which make evident the rigidity of the structures involved in the rigorous generalization of formula (8) to infinitedimensional case-c.f. Theorem 2.5. 
In Sect. 2, we introduce a regularization procedure through the projectors $Q_{n}$ to safely define a family of measures $v_{\phi, \psi}^{(N, n)}$ whose distributional limit $n \rightarrow+\infty$ permitted us to define the operator integral in Eq. (24)—c.f. Theorem 2.3. This may appear artificial at first glance and one may wonder if the regularization $Q_{n}$ can be removed. This is not the case-c.f. Sect. 4.1.

Moreover, even in the finite-dimensional case-where $Q_{n}$ can be chosen to be the identity and $v_{\phi, \psi}^{(N)}$ does exists as a complex measure on $\mathbb{R}^{2 N}$ - the properties of the sequence of measures $\left\{v_{\phi, \psi}^{(N)}\right\}_{N}$ are, however, not sufficient to define a suitable projective limit $N \rightarrow+\infty$-c.f. Sect. 4.2.

Finally, the convergence properties of $\mu_{\phi, \psi}^{(N, n)}$ as $n \rightarrow+\infty$ are considered in the best case scenario, namely when $P^{(A)}, P^{(B)}$ commute. Even in this, in a sense tamed situation, simple cases pop out where $\mu_{\phi, \psi}^{(N, n)}$ does not converge in the space of complex measure $\mathscr{M}\left(\mathbb{R}^{2 N}\right)$-c.f. Sect. 4.3 .

\subsection{The $Q_{n}$-regularization is generally necessary}

Lemma 2.2 introduces the regularized measure $v_{\phi, \psi}^{(N, n)}$ which is built out of the PVMs $P^{(A)}, P^{(B)}$ associated with the operators $A, B$ together with a suitable "regularizing" sequence of projectors $\left\{Q_{n}\right\}$. One may wonder whether it is possible to avoid the regularization $\left\{Q_{n}\right\}$, thus considering a complex measure $v_{\phi, \psi}^{(N)}$ defined on products of Borel set $I_{\ell}, J_{\ell} \in \mathscr{B}(\mathbb{R})$ as

$$
v_{\phi, \psi}^{(N)}\left(\times_{\ell=1}^{N} I_{\ell} \times J_{\ell}\right):=\left\langle\phi \mid P_{I_{1}}^{(A)} P_{J_{1}}^{(B)} \cdots P_{I_{N}}^{(A)} P_{J_{N}}^{(B)} \psi\right\rangle
$$

Unfortunately, $v_{\phi, \psi}^{(N)}$ generally does not extend to a complex measure on $\mathbb{R}^{2 N}$ as is in particular shown by the following physically relevant example.

Example 4.1 With reference to Example 3.3, let us check if, in this concrete case, $v_{\phi, \psi}^{(N)}$ extends to a complex measure on $\mathbb{R}^{2 N}$.

For all $I_{1}, J_{1}, \ldots, I_{N}, J_{N} \in \mathscr{B}(\mathbb{R})$, we have

$$
\begin{aligned}
v_{\phi, \psi}^{N}\left(\times_{\ell=1}^{N} I_{\ell} \times J_{\ell}\right) & =\int 1_{I_{1}}\left(x_{1}\right) 1_{J_{1}}\left(k_{1}\right) \cdots 1_{J_{N}}\left(k_{N}\right) \rho_{\phi, \psi}\left(x_{1}, k_{1}, \ldots, k_{N}\right) \mathrm{d} x_{1} \cdots \mathrm{d} k_{N}, \\
\rho_{\phi, \psi}\left(x_{1}, \ldots, k_{N}\right) & :=(2 \pi)^{-N+1 / 2} \overline{\phi\left(x_{1}\right)} \prod_{\ell=1}^{N-1} \mathrm{e}^{i k_{\ell}\left(x_{\ell+1}-x_{\ell}\right)} \mathrm{e}^{-i k_{N} x_{N}} \widehat{\psi}\left(k_{N}\right) .
\end{aligned}
$$

Since $\rho_{\phi, \psi} \in L_{\text {loc }}^{1}\left(\mathbb{R}^{2 N}, d^{N} x d^{N} k\right) \backslash L^{1}\left(\mathbb{R}^{2 N}, d^{N} x d^{N} k\right)$, it is evident that $v_{\phi, \psi}^{N}$ does not extend to a well-defined complex measure on $\mathbb{R}^{2 N}$ because the total variation of such complex measure would be $\left\|\rho_{\phi, \psi}\right\|_{L^{1}\left(\mathbb{R}^{2 N}, d^{N} x d^{N} k\right)}$ which is not finite. 


\subsection{The sequence of measures $v_{\phi, \psi}^{(N)}$ generally does not admit a (projective) limit}

Let us consider the elementary case of a finite-dimensional Hilbert space, where the regularization driven by $Q$ is not necessary. In fact, we can simply fix $Q_{n}=I$.

A natural issue is whether or not it is possible to interpret the limit for $N \rightarrow \infty$ on the right-hand side of (8) in terms of a integral on an infinite-dimensional space. Indeed, in the particular case where $\mathrm{H}$ is finite dimensional, for any $\phi, \psi \in \mathrm{H}$ it is possible to define a family of complex Borel measures $\left\{v_{\phi, \psi}^{(N)}\right\}_{N \in \mathbb{N}}$, with $v_{\phi, \psi}^{(N)}: \mathscr{B}\left(\mathbb{R}^{2 N}\right) \rightarrow \mathbb{C}$. By introducing for any $M, N \in \mathbb{N}$ with $M \leq N$, the projection maps $\pi_{M}^{N}: \mathbb{R}^{2 N} \rightarrow \mathbb{R}^{2 M}$, it is simple to see that the measures $\left\{v_{\phi, \psi}^{(N)}\right\}_{N \in \mathbb{N}}$ fulfill the consistency condition

$$
v_{\phi, \psi}^{(M)}=\left(\pi_{M}^{N}\right)_{*} v_{\phi, \psi}^{(N)}, \quad M \leq N,
$$

that is, for all $I_{1}, \ldots, I_{M}, J_{1}, \ldots, J_{M} \in \mathscr{B}(\mathbb{R})$

$$
v_{\phi, \psi}^{(M)}\left(\times_{\ell=1}^{M} I_{\ell} \times J_{\ell}\right)=v_{\phi, \psi}^{(N)}\left(\times_{\ell=1}^{M} I_{\ell} \times J_{\ell} \times(\mathbb{R} \times \mathbb{R})^{N-M}\right) .
$$

It is then meaningful to investigate whether the projective family of complex measures $\left\{v_{\phi, \psi}^{(N)}\right\}_{N \in \mathbb{N}}$ admits a projective limit. For that, let us consider the infinite-dimensional space $(\mathbb{R} \times \mathbb{R})^{\mathbb{N}}$ of all sequences $\left\{\left(\lambda_{n}, \mu_{n}\right)\right\}_{n \in \mathbb{N}}$. Moreover, let $\Sigma$ be the $\sigma$-algebra on $(\mathbb{R} \times \mathbb{R})^{\mathbb{N}}$ generated by the cylinder sets $\pi_{N}^{-1}(E)$, where $N \in \mathbb{N}, E \in \mathscr{B}\left(\mathbb{R}^{2 N}\right)$ and $\pi_{N}:(\mathbb{R} \times \mathbb{R})^{\mathbb{N}} \rightarrow(\mathbb{R} \times \mathbb{R})^{N}$ is the canonical projection map. Within this setting, it is meaningful to investigate the existence of a complex bounded measure $v_{\phi, \psi}$ on $\left((\mathbb{R} \times \mathbb{R})^{\mathbb{N}}, \Sigma\right)$ such that $v_{\phi, \psi}^{(N)}=\left(\pi_{N}\right)_{*} \nu_{\phi, \psi}$ for all $n \in \mathbb{N}$ (see "3" for further details). However, as proved in " 3 ," a necessary condition for the existence of $v_{\phi, \psi}$ is a uniform bound on the total variation $\left\|v_{\phi, \psi}^{(N)}\right\|=\left|v_{\phi, \psi}^{(N)}\left(\mathbb{R}^{2 N}\right)\right|$ of the measures $\left\{v_{\phi, \psi}^{(N)}\right\}_{N \in \mathbb{N}}$. Unfortunately, in general this does not hold when $A$ and $B$ do not commute as the following example shows.

Example 4.2 Let us consider the special case $\mathrm{H}=\mathbb{C}^{2}$, where $A:=\sigma_{z}$ and $B:=\sigma_{x}$, $\sigma_{z}, \sigma_{x}$ denoting two Pauli matrices. In this case, the Hilbert space is finite dimensional and for any $N \geq 1$ and $\phi, \psi \in \mathbb{C}^{2}$, it is possible to define the complex Borel measure $v_{\phi, \psi}^{N}$ on $\{ \pm 1\}^{2 N}$ (the topology being the discrete one) as:

$$
v_{\phi, \psi}^{N}\left(\left\{\left(a_{1}, b_{1}, \ldots, a_{N}, b_{N}\right)\right\}\right):=\left\langle\phi \mid P_{a_{1}}^{(A)} P_{b_{1}}^{(B)} \cdots P_{a_{N}}^{(A)} P_{b_{N}}^{(B)} \psi,\right\rangle
$$

where $a_{1}, \ldots, a_{N}, b_{1}, \ldots, b_{N} \in\{ \pm 1\}$ and $P_{ \pm}^{(A)}, P_{ \pm}^{(B)}$ are defined as

$$
P_{ \pm}^{(A)}:=\left|z_{ \pm}\right\rangle\left\langle z_{ \pm}\left|, \quad P_{ \pm}^{(B)}:=\right| x_{ \pm}\right\rangle\left\langle x_{ \pm}\right|
$$

where $\left|z_{+}\right\rangle,\left|z_{-}\right\rangle$, resp. $\left|x_{+}\right\rangle,\left|x_{-}\right\rangle$, are the normalized eigenvectors of $\sigma_{z}$, resp. $\sigma_{x}$.

The total variation of $v_{\phi, \psi}^{(N)}$ can be computed explicitly here. Indeed, by writing the vectors $\phi, \psi$ as linear combinations of the vectors of the orthonormal basis $\left\{\left|z_{+}\right\rangle,\left|z_{-}\right\rangle\right\}$ 
and $\left\{\left|x_{+}\right\rangle,\left|x_{-}\right\rangle\right\}$, namely:

$$
\phi=\alpha_{+}\left|z_{+}\right\rangle+\alpha_{-}\left|z_{-}\right\rangle, \quad \psi=\beta_{+}\left|x_{+}\right\rangle+\beta_{-}\left|x_{-}\right\rangle
$$

we get for any $\left(a_{1}, b_{1}, \ldots, a_{N}, b_{N}\right) \in\{ \pm 1\}^{2 N}$ :

$$
\left|v_{\phi, \psi}^{(N)}\left(\left\{\left(a_{1}, b_{1}, \ldots, a_{N}, b_{N}\right)\right\}\right)\right|=2^{-N+1 / 2}\left(\left|\alpha_{+}\right|+\left|\alpha_{-}\right|\right)\left(\left|\beta_{+}\right|+\left|\beta_{-}\right|\right) .
$$

Hence, we obtain

$$
\left|v_{\phi, \psi}^{(N)}\right|=\sqrt{2} 2^{N-2}\left(\left|\alpha_{+}\right|+\left|\alpha_{-}\right|\right)\left(\left|\beta_{+}\right|+\left|\beta_{-}\right|\right) .
$$

The sequence of measures $v_{\phi, \psi}^{(N)}$ cannot admit a projective limit, since $\sup _{N}\left|v_{\phi, \psi}^{(N)}\right|=$ $+\infty$.

The illustrated no-go result is strictly related to the non-commutativity of the spectral projectors $P^{(A)}$ and $P^{(B)}$. In fact, in the general case of a separable Hilbert space $\mathrm{H}$, if the spectral measures $P^{(A)}$ and $P^{(B)}$ commute then, for any couple of normalized vectors $\phi, \psi \in \mathrm{H}$ and for any $N \geq 1$, the formula

$$
v_{\phi, \psi}^{(N)}\left(\times_{\ell=1}^{N} I_{\ell} \times J_{\ell}\right)=\left\langle\phi \mid P_{I_{1}}^{(A)} P_{J_{1}}^{(B)} \cdots P_{I_{N}}^{(A)} P_{J_{N}}^{(B)} \psi\right\rangle
$$

defines a complex measure with finite total variation. In particular, since

$$
\left\langle\phi, P_{I_{1}}^{(A)} P_{J_{1}}^{(B)} \cdots P_{I_{N}}^{(A)} P_{J_{N}}^{(B)} \psi\right\rangle=\left\langle\phi \mid P_{\cap_{l=1}^{N} I_{l}}^{(A)} P_{\cap_{l=1}^{N} J_{l}}^{(B)} \psi\right\rangle,
$$

the following identity holds for any set $E \in \mathscr{B}\left(\mathbb{R}^{2 N}\right)$,

$$
v_{\phi, \psi}^{(N)}(E)=\int_{\mathbb{R}^{2}} 1_{E}\left(\lambda_{1}, \mu_{1}, \ldots \lambda_{1}, \mu_{1}\right) \mathrm{d} v_{\phi, \psi}^{1}\left(\lambda_{1}, \mu_{1}\right),
$$

where $v_{\phi, \psi}^{(1)}(I \times J)=\left\langle\phi \mid P_{I}^{(A)} P_{J}^{(B)} \psi\right\rangle$ is associated with the joint spectral measure of $A$ and $B$. By (60), it is simple to obtain the following inequality for any $N \geq 1$

$$
\left|v_{\phi, \psi}^{(N)}\right| \leq\left|v_{\phi, \psi}^{(1)}\right| \leq\|\phi\|\|\psi\| .
$$

In addition, in the particular case where $\phi=\psi$ and $\|\phi\|=1$ formula (8) admits a simple probabilistic interpretation. Indeed, in this case the measures $\left\{v_{\phi, \psi}^{(N)}\right\}_{N}$ form a consistent family of probability measures that can be interpreted as the joint distributions of a sequence $\left\{\xi_{n}, \eta_{n}\right\}_{n}$ of random variables with values in $\mathbb{R}^{2}$ which are in fact trivially completely correlated, i.e., $\left(\xi_{n}, \eta_{n}\right)=\left(\xi_{1}, \eta_{1}\right)$ for all $n \in \mathbb{N}$, and the distribution of the random vector $\left(\xi_{1}, \eta_{1}\right)$ is given by the joint spectral measure of $A$ and $B$.

In the case the commutativity condition is not fulfilled, then a uniform bound as (61) cannot be obtained in general as the following example shows. 
Example 4.3 Let us consider again $\mathrm{H}:=\mathbb{C}^{2}$ as before, and the two spectral measures $P^{(A)}$ and $P^{(B)}$ on $\{ \pm 1\}$ defined as

$$
P_{ \pm}^{(A)}:=\left|z_{ \pm}\right\rangle\left\langle z_{ \pm}\left|, \quad P_{ \pm}^{(B)}:=\right| v_{ \pm}\right\rangle\left\langle v_{ \pm}\right|
$$

where

$$
\left|v_{+}\right\rangle=(\cos \epsilon)\left|z_{+}\right\rangle+(\sin \epsilon)\left|z_{-}\right\rangle, \quad\left|v_{-}\right\rangle=-(\sin \epsilon)\left|z_{+}\right\rangle+(\cos \epsilon)\left|z_{-}\right\rangle, \quad \epsilon>0
$$

Let us choose $\phi:=\frac{\sqrt{2}}{2}\left|z_{+}\right\rangle+\frac{\sqrt{2}}{2}\left|z_{-}\right\rangle$and $\psi:=\frac{\sqrt{2}}{2}\left|v_{+}\right\rangle+\frac{\sqrt{2}}{2}\left|v_{-}\right\rangle$and consider again the complex measure $v_{\phi, \psi}^{(N)}$ on $\{ \pm 1\}^{2 N}$ defined for any $\left(a_{1}, b_{1}, \ldots, a_{N}, b_{N}\right) \in\{ \pm 1\}^{2 N}$ by

$$
v_{\phi, \psi}^{(N)}\left(\left\{\left(a_{1}, b_{1}, \ldots, a_{N}, b_{N}\right)\right\}\right):=\left\langle\phi \mid P_{a_{1}}^{(A)} P_{b_{1}}^{(B)} \cdots P_{a_{N}}^{(A)} P_{b_{N}}^{(B)} \psi\right\rangle .
$$

It is simple to verify that

$$
\begin{aligned}
\left|v_{\phi, \psi}^{(N)}\right| & =\sum_{\left(a_{1}, b_{1}, \ldots, a_{N}, b_{N}\right) \in\{ \pm 1\}^{2 N}}\left|v_{\phi, \psi}^{(N)}\left(\left\{\left(a_{1}, b_{1}, \ldots, a_{N}, b_{N}\right)\right\}\right)\right| \\
& =\frac{1}{2}(|\cos \epsilon|+|\sin \epsilon|)^{2 N-1}
\end{aligned}
$$

which shows that $\sup _{N}\left\|v_{\phi, \psi}^{(N)}\right\|=+\infty$, unless the spectral measures commute.

\subsection{Convergence of $\left\{\mu_{\phi, \psi}^{(N, n)}\right\}_{n \in \mathbb{N}}$ in $\mathscr{M}\left(\mathbb{R}^{2 N}\right)$ for commuting PVMs: a counterexample}

Let us consider once again the sequence of finite complex measure $v_{\phi, \psi}^{(N, n)}$ on $\mathbb{R}^{2 N}$. Since $Q_{n} \rightarrow I$, strongly it follows that for all $I_{\ell}, J_{\ell} \in \mathscr{B}\left(\mathbb{R}^{N}\right)$,

$$
\lim _{n \rightarrow+\infty} v_{\phi, \psi}^{(N, n)}\left(\times_{\ell=1}^{N} I_{\ell} \times J_{\ell}\right)=\left\langle\phi \mid P_{I_{1}}^{(A)} P_{J_{1}}^{(B)} \cdots P_{I_{N}}^{(A)} P_{J_{N}}^{(B)} \psi\right\rangle=: v_{\phi, \psi}^{(N)}\left(\times{ }_{\ell=1}^{N} I_{\ell} \times J_{\ell}\right)
$$

Unfortunately, as shown in Example 4.1, $v_{\phi, \psi}^{(N)}$ does not define a measure on $\mathbb{R}^{2 N}$. However, whenever $\left[P^{(A)}, P^{(B)}\right]=0, v_{\phi, \psi}^{(N)}$ does define a complex measure on $\mathbb{R}^{2 N}$ c.f. Sect. 4.2. Therefore, one may wonder whether the convergence $v_{\phi, \psi}^{(N, n)} \rightarrow v_{\phi, \psi}^{(N)}$ holds true in the space of complex measure $\mathscr{M}\left(\mathbb{R}^{2 N}\right)$, namely if

$$
\lim _{n \rightarrow+\infty}\left\|v_{\phi, \psi}^{(N, n)}-v_{\phi, \psi}^{(N)}\right\|=\lim _{n \rightarrow+\infty}\left|v_{\phi, \psi}^{(N, n)}-v_{\phi, \psi}^{(N)}\right|\left(\mathbb{R}^{2 N}\right)=0
$$

Unfortunately, this is not the case as shown in particular by the following example. 
Example 4.4 Let us consider $\mathrm{H}=L^{2}([0,1], \mathrm{d} x)$ and let $A=B=X$ be the usual position operator over $[0,1]-$ c.f. Example 4.1 . We consider the complete orthonormal basis $\left\{e_{k}\right\}_{k \in \mathbb{Z}}$ of $L^{2}([0,1], \mathrm{d} x)$ made by exponentials $e_{k}(x):=\mathrm{e}^{2 \pi i k x}$ and we set $Q_{n}:=\sum_{|k| \leq n}\left|e_{k}\right\rangle\left\langle e_{k}\right|$ for $n \in \mathbb{N}$. Moreover, we choose $\psi=\phi=1_{[0,1]} \in$ $L^{2}([0,1], \mathrm{d} x)$. It follows that for all $I, J \in \mathscr{B}([0,1]$ ) (where the Borel algebra is referred to the topology on $[0,1]$ form $\mathbb{R}$ ),

$$
\begin{aligned}
& v_{\phi, \psi}^{(1)}(I \times J)=\left\langle\phi \mid P_{I \cap J}^{(X)} \psi\right\rangle=\int_{0}^{1} 1_{I \cap J}(x) \mathrm{d} x, \\
& v_{\phi, \psi}^{(1, n)}(I \times J)=\left\langle\phi \mid P_{I}^{(X)} Q_{n} P_{J}^{(X)} \psi\right\rangle=\int_{0}^{1} \int_{0}^{1} 1_{I}(x) 1_{J}(y) \rho_{n}(x-y) \mathrm{d} x \mathrm{~d} y,
\end{aligned}
$$

where $\rho_{n}(x-y):=\sum_{|k| \leq n} e_{k}(x-y)$. A direct computation yields $\left\|v_{\phi, \psi}^{(1)}\right\|=$ $\left|v_{\phi, \psi}^{(1)}\right|\left([0,1]^{2}\right)=1$ while $\left\|v_{\phi, \psi}^{(1, n)}\right\|=\left\|\rho_{n}\right\|_{L^{1}([0,1], \mathrm{d} x)}$. Since the linear map $L^{1}([0,1], \mathrm{d} x) \ni f \mapsto m_{f} \in \mathscr{M}\left([0,1]^{2}\right)$ —efined by $m_{f}(E):=\int_{E} f(x-y) \mathrm{d} x \mathrm{~d} y$ is a (non-surjective) isometry and both spaces are complete, it follows that convergence of $v_{\psi, \psi}^{(1, N)}$ in $\mathscr{M}\left([0,1]^{2}\right)$ would imply convergence of $\rho_{n}$ in $L^{1}([0,1], \mathrm{d} x)$ to some function therein. However, this is impossible since $\rho_{n}$ weakly converges to the Dirac delta $\delta$ centered at 0 which cannot be represented by a function of $L^{1}([0,1], \mathrm{d} x)$.

\section{Toward a physical interpretation}

In the particular case where the Hilbert space $\mathrm{H}$ is finite dimensional and no technical issues must be addressed, it is interesting to discuss the physical interpretations of formula (8).

To this end, we recall that if, as assumed, the Hilbert space is separable with $\operatorname{dim}(H) \neq 2$, the Gleason theorem $[8,11]$ establishes that quantum states viewed as $\sigma$-additive measures $\mu$ on $\mathcal{L}(\mathrm{H})$ are one-to-one with positive, trace-class, unit-trace operators $\rho_{\mu}: \mathrm{H} \rightarrow \mathrm{H}$. Pure states are the extremal elements $v$ of the convex body of states and they are in a one-to-one correspondence with unit vectors $\psi_{\nu}$ up to phases and $v(P)=\left\|P \psi_{v}\right\|^{2}$ is the probability that $P \in \mathscr{L}(\mathrm{H})$ is true if the pure state is represented by $\psi_{\nu}$.

\subsection{Non-commutativity and quantum interference terms}

We start the analysis with the following elementary observation. Since the identity operator can be decomposed either as $I=\sum_{\lambda \in \sigma(A)} P_{\lambda}^{(A)}$ or as $I=\sum_{\mu \in \sigma(B)} P_{\mu}^{(B)}$, for any $N \in \mathbb{N}$ and $\psi \in \mathrm{H}$ we have

$$
\psi=\sum_{\substack{\lambda_{1}, \ldots, \lambda_{N} \in \sigma(A) \\ \mu_{1}, \ldots, \mu_{N} \in \sigma(B)}} P_{\lambda_{1}}^{(A)} P_{\mu_{1}}^{(B)} \cdots P_{\lambda_{N}}^{(A)} P_{\mu_{N}}^{(B)} \psi
$$


Above, the state vector $\psi$ is decomposed into a coherent superposition of generally non-mutually orthogonal vector states. These vectors would be mutually orthogonal if the PVMs commuted. The presence in (8) of these non-orthogonal vectors is the source of a quantum interference phenomenon we are going to illustrate.

The weak version of formula (8) reads as follows:

$$
\begin{aligned}
& \langle\phi \mid f(a A+b B) \psi\rangle \\
& =\lim _{N \rightarrow+\infty} \sum_{\substack{\lambda_{1}, \ldots, \lambda_{N} \in \sigma(A) \\
\mu_{1}, \ldots, \mu_{N} \in \sigma(B)}} f\left(\frac{1}{N} \sum_{j=1}^{N}\left(a \lambda_{j}+b \mu_{j}\right)\right)\left\langle\phi \mid P_{\lambda_{1}}^{(A)} P_{\mu_{1}}^{(B)} \cdots P_{\lambda_{N}}^{(A)} P_{\mu_{N}}^{(B)} \psi\right\rangle,
\end{aligned}
$$

for any $\phi, \psi \in \mathrm{H}$. In particular, when $\phi=\psi$, we get

$$
\begin{aligned}
& \langle\psi \mid f(a A+b B) \psi\rangle \\
& =\lim _{N \rightarrow+\infty} \sum_{\substack{\lambda_{1}, \ldots, \lambda_{N} \in \sigma(A) \\
\mu_{1}, \ldots, \mu_{N} \in \sigma(B)}} f\left(\frac{1}{N} \sum_{j=1}^{N}\left(a \lambda_{j}+b \mu_{j}\right)\right)\left\langle\psi \mid P_{\lambda_{1}}^{(A)} P_{\mu_{1}}^{(B)} \cdots P_{\lambda_{N}}^{(A)} P_{\mu_{N}}^{(B)} \psi\right\rangle .
\end{aligned}
$$

As usual, the left-hand side coincides with the expectation value of the observable $f(a A+b B)$ when the state is pure and represented by the unit vector $\psi$. In particular, if $f=1_{[\alpha, \beta)}$ [in this case (64) should be replaced by (14)], the left-hand side is nothing but the probability of obtaining an outcome in $[\alpha, \beta)$ when measuring $a A+b B$ over the pure state represented by the unit vector $\psi$.

Exploiting (62) in the expansion the left entry of the inner products, formula (64) can be rephrased to

$$
\begin{aligned}
& \langle\psi \mid f(a A+b B) \psi\rangle \\
& =\lim _{N \rightarrow+\infty} \sum_{\substack{\lambda_{1}, \ldots, \lambda_{N} \in \sigma(A) \\
\mu_{1}, \ldots, \mu_{N} \in \sigma(B)}} f\left(\frac{1}{N} \sum_{j=1}^{N}\left(a \lambda_{j}+b \mu_{j}\right)\right) \\
& \sum_{\substack{\tilde{\lambda}_{1}, \ldots, \tilde{\lambda}_{N} \in \sigma(A) \\
\tilde{\mu}_{1}, \ldots, \tilde{\mu}_{N} \in \sigma(B)}}\left\langle P_{\tilde{\lambda}_{1}}^{(A)} P_{\tilde{\mu}_{1}}^{(B)} \cdots P_{\tilde{\lambda}_{N}}^{(A)} P_{\tilde{\mu}_{N}}^{(B)} \psi \mid P_{\lambda_{1}}^{(A)} P_{\mu_{1}}^{(B)} \cdots P_{\lambda_{N}}^{(A)} P_{\mu_{N}}^{(B)} \psi\right\rangle, \\
& =\lim _{N \rightarrow+\infty} \sum_{\substack{\lambda_{1}, \ldots, \lambda_{N} \in \sigma(A) \\
\mu_{1}, \ldots, \mu_{N} \in \sigma(B)}} f\left(\frac{1}{N} \sum_{j=1}^{N}\left(a \lambda_{j}+b \mu_{j}\right)\right)\left\|P_{\lambda_{1}}^{(A)} P_{\mu_{1}}^{(B)} \cdots P_{\lambda_{N}}^{(A)} P_{\mu_{N}}^{(B)} \psi\right\|^{2}+
\end{aligned}
$$




$$
\begin{aligned}
& +\lim _{N \rightarrow+\infty} \sum_{\substack{\lambda_{1}, \ldots, \lambda_{N} \in \sigma(A) \\
\mu_{1}, \ldots, \mu_{N} \in \sigma(B)}} f\left(\frac{1}{N} \sum_{j=1}^{N}\left(a \lambda_{j}+b \mu_{j}\right)\right) \\
& \quad \sum_{\substack{\tilde{\lambda}_{1}, \ldots, \tilde{\lambda}_{N} \in \sigma(A) \\
\tilde{\mu}_{1}, \ldots, \tilde{\mu}_{N} \in \sigma(B),(\tilde{\tilde{\lambda}}, \tilde{\tilde{\mu}}) \neq(\bar{\lambda}, \bar{\mu})}}\left\langle P_{\tilde{\lambda}_{1}}^{(A)} P_{\tilde{\mu}_{1}}^{(B)} \cdots P_{\tilde{\lambda}_{N}}^{(A)} P_{\tilde{\mu}_{N}}^{(B)} \psi \mid P_{\lambda_{1}}^{(A)} P_{\mu_{1}}^{(B)} \cdots P_{\lambda_{N}}^{(A)} P_{\mu_{N}}^{(B)} \psi\right\rangle, \\
&
\end{aligned}
$$

where we defined $(\bar{\lambda}, \bar{\mu}):=\left(\lambda_{1}, \ldots, \lambda_{N}, \mu_{1}, \ldots, \mu_{N}\right)$ and $(\overline{\tilde{\lambda}}, \overline{\tilde{\mu}}):=\left(\tilde{\lambda}_{1}, \ldots, \tilde{\lambda}_{N}, \tilde{\mu}_{1}\right.$, $\left.\ldots, \tilde{\mu}_{N}\right)$.

The considered expectation value is therefore tantamount to the sum two distinct kinds of terms.

(1) The first type admits to some extent (see (1) Remark 5.1) a "classical" probabilistic interpretation relying on a recursive use of Born's rule and the standard Lüdersvon Neumann's post-measurement state postulate (see, e.g., [4,18,25]). As is well known, the former postulate states that if the initial pure states are represented by the unit vector $\psi \in \mathrm{H}$, the probability that the outcome of an elementary YES-NO observable $P \in \mathscr{L}(\mathrm{H})$ is 1 (YES) is $\|P \psi\|^{2}$. The latter postulate says that if the outcome resulted to be 1 , then the post-measurement state is represented by the normalized vector $\|P \psi\|^{-1} P \psi$. As a matter of fact, the positive number

$$
\left\|P_{\lambda_{1}}^{(A)} P_{\mu_{1}}^{(B)} \cdots P_{\lambda_{N}}^{(A)} P_{\mu_{N}}^{(B)} \psi\right\|^{2} \in[0,1]
$$

can be interpreted as a conditional probability ${ }^{7}$ : the probability that, given the initial pure state of the system described by the normalized vector $\psi \in \mathrm{H}$, in a sequence of $2 N$ measurements of observables $O_{j}, J=1, \ldots, 2 N$, with $O_{j}=B$ for $j$ odd and $O_{j}=A$ for $j$ even, the sequence of outcomes is exactly $\left(\mu_{N}, \lambda_{N}, \ldots, \mu_{1}, \lambda_{1}\right)$. Let us illustrate this point in some detail. Given a general observable $O$ in our finite-dimensional Hilbert space with (necessarily discrete) spectrum $\sigma(O)$, and a normalized vector $\phi \in \mathrm{H}$, we shall denote with the symbol $\mathbb{P}(O=o \mid \phi)$ the probability that, given that the (pure) state of the system is represented by $\phi$, the outcome of the measurement of $O$ is the real number $o \in \sigma(O)$, namely

$$
\mathbb{P}(O=o \mid \phi)=\left\|P_{o}^{(O)} \phi\right\|^{2},
$$

where $P_{o}^{(O)}$ is the spectral projector associated with the eigenvalue $o \in \sigma(O)$. Let us denote by $\psi_{\lambda_{j}, \mu_{j}, \lambda_{j+1}, \ldots, \lambda_{N}, \mu_{N}}$ and $\psi_{\mu_{j}, \lambda_{j+1}, \mu_{j+1}, \ldots, \lambda_{N}, \mu_{N}}$ the post-measurement normalized vectors

\footnotetext{
7 The general notion of quantum conditional probability is still an open and controversial topic of the quantum theory $[10,14,31]$. Our discussion will, however, involve very basic and straightforward arguments.
} 


$$
\begin{aligned}
& \psi_{\lambda_{j}, \mu_{j}, \lambda_{j+1}, \ldots, \lambda_{N}, \mu_{N}}:=\frac{P_{\lambda_{j}}^{(A)} P_{\mu_{j}}^{(B)} P_{\lambda_{j+1}}^{(A)} \cdots P_{\lambda_{N}}^{(A)} P_{\mu_{N}}^{(B)} \psi}{\left\|P_{\lambda_{j}}^{(A)} P_{\mu_{j}}^{(B)} P_{\lambda_{j+1}}^{(A)} \cdots P_{\lambda_{N}}^{(A)} P_{\mu_{N}}^{(B)} \psi\right\|}, \\
& \psi_{\mu_{j}, \lambda_{j+1}, \mu_{j+1}, \ldots, \lambda_{N}, \mu_{N}}:=\frac{P_{\mu_{j}}^{(B)} P_{\lambda_{j+1}}^{(A)} P_{\mu_{j+1}}^{(A)} \cdots P_{\lambda_{N}}^{(A)} P_{\mu_{N}}^{(B)} \psi}{\left\|P_{\mu_{j}}^{(B)} P_{\lambda_{j+1}}^{(A)} P_{\mu_{j+1}}^{(A)} \cdots P_{\lambda_{N}}^{(A)} P_{\mu_{N}}^{(B)} \psi\right\|},
\end{aligned}
$$

with $j=1, \ldots, N$. We then have:

$$
\begin{aligned}
& \left\|P_{\lambda_{1}}^{(A)} P_{\mu_{1}}^{(B)} \cdots P_{\lambda_{N}}^{(A)} P_{\mu_{N}}^{(B)} \psi\right\|^{2}=\mathbb{P}\left(A=\lambda_{1} \mid \psi_{\mu_{1}, \lambda_{2}, \ldots, \lambda_{N}, \mu_{N}}\right)\left\|P_{\mu_{1}}^{(B)} \cdots P_{\lambda_{N}}^{(A)} P_{\mu_{N}}^{(B)} \psi\right\|^{2} \\
& \quad=\mathbb{P}\left(A=\lambda_{1} \mid \psi_{\mu_{1}, \lambda_{2}, \ldots, \lambda_{N}, \mu_{N}}\right) \mathbb{P}\left(B=\mu_{1} \mid \psi_{\lambda_{2}, \mu_{2}, \ldots, \lambda_{N}, \mu_{N}}\right)\left\|P_{\lambda_{2}}^{(A)} P_{\mu_{2}}^{(B)} \cdots P_{\lambda_{N}}^{(A)} P_{\mu_{N}}^{(B)} \psi\right\|^{2} .
\end{aligned}
$$

By induction over $N$, we get the wanted result:

$$
\begin{aligned}
& \left\|P_{\lambda_{1}}^{(A)} P_{\mu_{1}}^{(B)} \cdots P_{\lambda_{N}}^{(A)} P_{\mu_{N}}^{(B)} \psi\right\|^{2} \\
& =\Pi_{j=1}^{N-1} \mathbb{P}\left(A=\lambda_{j} \mid \psi_{\mu_{j}, \lambda_{j+1}, \ldots, \lambda_{N}, \mu_{N}}\right) \mathbb{P}\left(B=\mu_{j} \mid \psi_{\lambda_{j+1}, \mu_{j+1}, \ldots, \lambda_{N}, \mu_{N}}\right) \\
& \quad \cdot \mathbb{P}\left(A=\lambda_{N} \mid \psi_{\mu_{N}}\right) \mathbb{P}\left(B=\mu_{N} \mid \psi\right)
\end{aligned}
$$

It is now evident that the right-hand side has the natural interpretation as the probability that, given the initial pure state of the system described by the normalized vector $\psi \in \mathrm{H}$, in a sequence of $2 N$ measurements of observables $O_{j}$, $J=1, \ldots, 2 N$, with $O_{j}=B$ for $j$ odd and $O_{j}=A$ for $j$ even, the sequence of outcomes is exactly $\left(\mu_{N}, \lambda_{N}, \ldots, \mu_{1}, \lambda_{1}\right)$.

(2) The remaining terms appearing in the last line of Eq. (65) are of the form

$$
\left\langle P_{\tilde{\lambda}_{1}}^{(A)} P_{\tilde{\mu}_{1}}^{(B)} \cdots P_{\tilde{\lambda}_{N}}^{(A)} P_{\tilde{\mu}_{N}}^{(B)} \psi \mid P_{\lambda_{1}}^{(A)} P_{\mu_{1}}^{(B)} \cdots P_{\lambda_{N}}^{(A)} P_{\mu_{N}}^{(B)} \psi\right\rangle,
$$

with $\left(\tilde{\lambda}_{1}, \ldots, \tilde{\mu}_{N}\right) \neq\left(\lambda_{1}, \ldots, \mu_{N}\right)$ being complex numbers which can be interpreted as quantum amplitudes or interference terms arising from the coherent decomposition (62) into non-orthogonal addends. In the trivial case where the spectral measures $\left\{P_{\lambda}^{(A)}\right\}_{\lambda \in \sigma(A)}$ and $\left\{P_{\mu}^{(B)}\right\}_{\mu \in \sigma(B)}$ commute, it is easy to check that all the interference terms (67) vanish as already remarked. In this sense, their presence has an evident quantum nature.

Remark 5.1 (1) It is worth stressing that also the quantum conditional probability discussed in (1) enjoys different properties than those of the classical conditional probability when the PVMs do not commute. This is because we are here dealing with the quantum notion of probability characterized by Gleason's theorem, defined on a orthomodular lattice rather than a $\sigma$-algebra. In particular, the classical identity $\mathbb{P}(U \mid V) \mathbb{P}(V)=\mathbb{P}(V \mid U) \mathbb{P}(U)$ has not a corresponding version at quantum level when $U$ and $V$ are replaced for quantum incompatible events. This is reflected from the fact that the order in the sequence of measurements $P_{\mu_{1}}^{(B)} \cdots P_{\lambda_{N}}^{(A)} P_{\mu_{N}}^{(B)}$ cannot be ignored. 
(2) If $\mathrm{H}$ is finite dimensional, we have the following limit in the operator norm

$$
\begin{aligned}
f(A+B) & =\lim _{N \rightarrow+\infty} f_{N}(A, B) \\
& =\lim _{N \rightarrow+\infty} \sum_{\substack{\lambda_{1}, \ldots, \lambda_{N} \in \sigma(A) \\
\mu_{1}, \ldots, \mu_{N} \in \sigma(B)}} f\left(\frac{1}{N} \sum_{j=1}^{N}\left(\lambda_{j}+\mu_{j}\right)\right) P_{\lambda_{1}}^{(A)} P_{\mu_{1}}^{(B)} \cdots P_{\lambda_{N}}^{(A)} P_{\mu_{N}}^{(B)} .
\end{aligned}
$$

We now provide an expansion of $f_{N}(A, B)$ in terms of spectral commutators of $A$ and $B$ which highlights the link between non-commutativity of spectral measures and appearance of terms of second kind in the right-hand side of (65). For that let $C_{\lambda, \mu}:=$ $\left[P_{\lambda}^{(A)}, P_{\mu}^{(B)}\right]$ and set $\wp_{N}\left(\lambda_{1}, \ldots, \lambda_{N} ; \mu_{1}, \ldots, \mu_{N}\right):=P_{\lambda_{1}}^{(A)} P_{\mu_{1}}^{(B)} \cdots P_{\lambda_{N}}^{(A)} P_{\mu_{N}}^{(B)}$. A direct computation leads to

$$
\begin{aligned}
\wp_{N}\left(\lambda_{1}, \ldots, \lambda_{N} ; \mu_{1}, \ldots, \mu_{N}\right) & :=P_{\lambda_{1}}^{(A)} P_{\mu_{1}}^{(B)} \cdots P_{\lambda_{N}}^{(A)} P_{\mu_{N}}^{(B)} \\
& =\left(P_{\mu_{1}}^{(B)} \delta_{\lambda_{1}, \lambda_{2}}+C_{\lambda_{1}, \mu_{1}}\right) P_{\lambda_{2}}^{(A)} P_{\mu_{2}}^{(B)} \cdots P_{\lambda_{N}}^{(A)} P_{\mu_{N}}^{(B)} \\
& =\left(P_{\mu_{1}}^{(B)} \delta_{\lambda_{1}, \lambda_{2}}+C_{\lambda_{1}, \mu_{1}}\right) \wp_{N-1}\left(\lambda_{2}, \ldots, \lambda_{N} ; \mu_{2}, \ldots, \mu_{N}\right) \\
& =\prod_{\ell=1}^{N}\left(P_{\mu_{\ell}}^{(B)} \Delta_{\lambda_{\ell}, \lambda_{\ell+1}}+C_{\lambda_{\ell}, \mu_{\ell}}\right),
\end{aligned}
$$

where we set $\Delta_{\lambda_{\ell}, \lambda_{\ell+1}}=\delta_{\lambda_{\ell}, \lambda_{\ell+1}}$ for $1 \leq \ell<N, \delta_{i, j}$ denoting the Kronecker symbol, while $\Delta_{\lambda_{N}, \lambda_{N+1}}=P_{\lambda_{N}}^{(A)}$. Notice that the product of the terms $P_{\mu_{\ell}}^{(B)} \Delta_{\lambda_{\ell}, \lambda_{\ell+1}}$ provides the contribution arising from the classical convolution formula, namely $\prod_{\ell=1}^{N} P_{\mu_{\ell}}^{(B)} \Delta_{\lambda_{\ell}, \lambda_{\ell+1}}=\prod_{\ell=1}^{N-1} \delta_{\lambda_{\ell}, \lambda_{\ell+1}} \delta_{\mu_{\ell}, \mu_{\ell+1}} P_{\lambda_{1}}^{(A)} P_{\mu_{1}}^{(B)}$. Products containing a commutator factor $C_{\lambda, \mu}$ represent a "perturbative" quantum correction to the commutative formula. Writing the product of spectral projectors as a sum over insertion of commutators, we obtain a "perturbative" expansion of $f(A+B)$ whose first terms are given by

$$
\begin{aligned}
f(A+B) & =\sum_{\substack{\lambda \in \sigma(A) \\
\mu \in \sigma(B)}} f(\lambda+\mu) P_{\lambda}^{(B)} P_{\mu}^{(A)} \\
& +\lim _{N \rightarrow+\infty} \sum_{\substack{\lambda_{1}, \lambda_{2} \in \sigma(A) \\
\mu_{1}, \mu_{2} \in \sigma(B)}} f\left[\frac{1}{N}\left(\lambda_{1}+(N-1) \lambda_{2}+\mu_{1}+(N-1) \mu_{2}\right)\right] C_{\lambda_{1}, \mu_{1}} P_{\mu_{2}}^{(B)} P_{\lambda_{2}}^{(A)} \\
& +\lim _{N \rightarrow+\infty} \sum_{\substack{\lambda_{1}, \lambda_{2} \in \sigma(A) \\
\mu_{1}, \mu_{2} \in \sigma(B)}} f\left[\frac{1}{N}\left((N-1) \lambda_{1}+\lambda_{2}+(N-1) \mu_{1}+\mu_{2}\right)\right] P_{\mu_{1}}^{(B)} C_{\lambda_{2}, \mu_{2}}
\end{aligned}
$$




$$
\begin{aligned}
& +\lim _{N \rightarrow+\infty} \sum_{\substack{\lambda_{1}, \lambda_{2} \in \sigma(A) \\
\mu_{1}, \mu_{2}, \mu_{3} \in \sigma(B)}} \sum_{k=2}^{N-1} f\left[\frac{1}{N}\left((k-1) \mu_{1}+\mu_{2}+(N-k) \mu_{3}+k \lambda_{1}+(N-k) \lambda_{2}\right)\right] \\
& \cdot P_{\mu_{1}}^{(B)} C_{\lambda_{1}, \mu_{2}} P_{\mu_{3}}^{(B)} P_{\lambda_{2}}^{(A)}+\cdots
\end{aligned}
$$

\subsection{Sum over all possible histories}

From now on, we assume $a=b=1$ for the sake of simplicity, still sticking to the case of a finite-dimensional Hilbert space $\mathrm{H}$. Formula (8) allows us to construct (weakly) the spectral measure of $A+B$ in terms of a particular limiting procedure. Let us consider, for any $N \in \mathbb{N}$ the finite sequence of $2 N$ observables $O_{1}, \ldots, O_{2 N}$, where for $j=1, \ldots, N O_{2 j}=A$ and $O_{2 j-1}=B$. It corresponds to a sequence of alternating measurements of the couple of observables $A$ and $B$. As discussed above, the statistics of these sequences of measurements is related to the complex measures $v_{\phi, \psi}^{(A, B, \ldots, A, B)}$ on $\sigma(A) \times \cdots \times \sigma(B)$ defined as

$$
v_{\phi, \psi}^{(A, B, \ldots, A, B)}\left(I_{1} \times J_{1} \times \cdots I_{N} \times J_{N}\right)=\left\langle\phi \mid P_{I_{1}}^{(A)} P_{J_{1}}^{(B)} \cdots P_{I_{N}}^{(A)} P_{J_{N}}^{(B)} \psi\right\rangle .
$$

We can in this way associate with any observable $O_{n}$ a map $T\left(O_{n}\right)$ on $\Omega:=(\sigma(A) \times$ $\sigma(B))^{\mathbb{N}}$ as follows,

$$
T\left(O_{2 j}\right)\left(\lambda_{1}, \mu_{1}, \ldots, \lambda_{n}, \mu_{n}, \ldots\right)=\lambda_{j}, \quad T\left(O_{2 j-1}\right)\left(\lambda_{1}, \mu_{1}, \ldots, \lambda_{n}, \mu_{n}, \ldots\right)=\mu_{j}, \quad j \geq 1 .
$$

We shall henceforth set $T\left(O_{2 j}\right) \equiv \xi_{j}$ and $T\left(O_{2 j-1}\right) \equiv \eta_{j}, j \geq 1$ to shorten the notation. Formally, we have for any $N \geq 1$ and $f_{1}, \ldots, f_{N}, g_{1}, \ldots, g_{N} \in C_{b}(\mathbb{R})$

$$
\left\langle\phi \mid \Pi_{j=1}^{N} f_{j}(A) g_{j}(B) \psi\right\rangle=\mathbb{E}\left[\Pi_{j=1}^{N} f_{j}\left(\xi_{j}\right) g_{j}\left(\eta_{j}\right)\right]
$$

where the expectation value on the right-hand side of (68) stands for the integral

$$
\int_{\sigma(A) \times \cdots \times \sigma(B)} \Pi_{j=1}^{N} f_{j}\left(\lambda_{j}\right) g_{j}\left(\mu_{j}\right) \mathrm{d} v_{\phi, \psi}^{(A, B, \ldots, A, B)}\left(\lambda_{1}, \ldots, \mu_{N}\right) .
$$

Referring to a measure space $(\Omega, \mathscr{F}, v)$, with $\Omega=(\sigma(A) \times \sigma(B))^{\mathbb{N}}, \mathscr{F}$ the $\sigma$ algebra generated by the cylinder sets (see "3") and $v$ a sigma additive measure on $\mathscr{F}$ with finite total variation (see Sect. 3.2), the sets $\left\{\xi_{n}\right\}_{n \in \mathbb{N}}$ and $\left\{\eta_{n}\right\}_{n \in \mathbb{N}}$ cannot in general be regarded as sequences of random variables (i.e., measurable functions) thereon, where $v$ extends $v_{\phi, \psi}^{(A, B, \ldots, A, B)}$. However, for any finite $N$ the integral (69) is well defined and $\left\{\xi_{n}\right\}_{n \in \mathbb{N}}$ and $\left\{\eta_{n}\right\}_{n \in \mathbb{N}}$ are called pseudo-stochastic process. They find applications, e.g., in the mathematical definition of Feynman path integrals as well as in the construction of Feynman-Kac-type formulae for PDEs that do not satisfy maximum principle [2]. 
In this context, the construction so far defined describes the spectral measure of $A+B$ in terms of a particular limiting procedure. Referring to the sequence of pseudorandom variables $\left\{\zeta_{n}\right\}_{n \in \mathbb{N}}$ defined as $\zeta_{n}:=\xi_{n}+\eta_{n}$, for $N \geq 1$ let $S_{N}$ denote the map $S_{N}:=\sum_{n=1}^{N} \zeta_{n}$. Then, according to formula (8), for any function $f \in \mathscr{F}(\mathbb{R})$ (or also $f \in C^{\infty}(\mathbb{R})$ since here $A, B \in \mathfrak{B}(\mathrm{H})$ automatically because $\left.\operatorname{dim} \mathrm{H}<+\infty\right)$, it holds

$$
\langle\phi \mid f(A+B) \psi\rangle=\lim _{N \rightarrow+\infty} \mathbb{E}\left[f\left(\frac{S_{N}}{N}\right)\right],
$$

which can be interpreted as a weak convergence of the (complex) distribution of the arithmetic mean of the first $N$ pseudorandom variables $\zeta_{n}$ to the measure $\mathscr{B}(\mathbb{R}) \ni$ $E \mapsto\left\langle\phi \mid P_{E}^{(A+B)} \psi\right\rangle$. Since we are dealing with discrete spectra, the formula above can be rephrased to

$$
\begin{aligned}
& \langle\phi \mid f(A+B) \psi\rangle \\
& =\lim _{N \rightarrow+\infty} \sum_{\substack{\lambda_{1}, \ldots, \lambda_{N} \in \sigma(A) \\
\mu_{1}, \ldots, \mu_{N} \in \sigma(B)}} f\left(\frac{1}{N} \sum_{n=1}^{N}\left(\lambda_{n}+\mu_{n}\right)\right) v_{\phi, \psi}^{(A, B, \ldots, A, B)}\left(\left\{\lambda_{1}, \mu_{1}, \ldots, \lambda_{N}, \mu_{N}\right\}\right)
\end{aligned}
$$

and the right-hand side can be interpreted as a "sum over all possible histories." In other words, that is an integral over the set of all possible outcomes of a sequence of consecutive measurements of the observables $A$ and $B$. It is worth stressing that the same result can be obtained by means of a different construction, by considering the family of complex measures $v_{\phi, \psi}^{(B, A, \ldots, B, A)}$ instead of $v_{\phi, \psi}^{(A, B, \ldots, A, B)}$, describing a sequence of consecutive measurement of the observables $A$ and $B$ which starts with the measurement of $A$.

\section{Summary}

This work tackles the issue of connecting the spectral measure $P^{(a A+b B)}$ of a linear combination of two observables $A, B$ with the spectral measures $P^{(A)}, P^{(B)}$ of $A$ and $B$, in the general case of a pair of non-compatible observables. This problem is relevant from the physical point of view as it helps in justifying the linear structure of the space of observables, which is assumed in the standard formulation of Quantum Theory. As a matter of fact, in Sect. 2 we establish a new formula (32), relating suitable functions $f(a A+b B)$ of two generally non-commuting and unbounded observables $A$ and $B$ with their PVMs $P^{(A)}$ and $P^{(B)}$. That formula is the central achievement of this paper expressed by Theorem 2.5, whose proof relies essentially on Trotter formula. This constrains the class of functions $f$ admitted by (32) - in Sect. 4 we show that the hypothesis necessary to establish our main result cannot be relaxed-however, this class of functions is nevertheless sufficiently large to allow the application of (32) to various cases of physical relevance discussed in Sect. 3. In fact, our formula has a nice interplay with the structure of Jordan algebra of observables and also with the notion of Feynman integral and the Feynman-Kac formula since, essentially, it specializes 
to them when $A$ and $B$ are suitably chosen. In addition, identity (32) has an appealing aspect from the physical viewpoint as it contains some terms which are suitable for some operational physical interpretation, as discussed in Sect. 5.

Acknowledgements The authors are grateful to C. Fewster, R. Ghiloni and I. Khavkine for useful discussions. The authors also thank an anonymous reviewer for his/her detailed and careful suggestions to improve the text and for having pointed out further relevant bibliography.

Funding Open access funding provided by Università degli Studi di Trento within the CRUI-CARE Agreement.

Open Access This article is licensed under a Creative Commons Attribution 4.0 International License, which permits use, sharing, adaptation, distribution and reproduction in any medium or format, as long as you give appropriate credit to the original author(s) and the source, provide a link to the Creative Commons licence, and indicate if changes were made. The images or other third party material in this article are included in the article's Creative Commons licence, unless indicated otherwise in a credit line to the material. If material is not included in the article's Creative Commons licence and your intended use is not permitted by statutory regulation or exceeds the permitted use, you will need to obtain permission directly from the copyright holder. To view a copy of this licence, visit http://creativecommons.org/licenses/by/4.0/.

\section{Appendix A: Proof of some propositions}

Proof of Lemma 2.2. (a) First of all, let us prove that there is a complex Borel measure on $\mathbb{R}^{2 N}$ (with finite total variation according to Sect. 1) satisfying (21). Notice that if such a measure exists, it must be unique in view of [5, Theorem 3.3]. Let $\left\{h_{k}\right\}_{k=1, \ldots, d_{n}}$ be an orthonormal basis of the finite-dimensional subspace $Q_{n}(\mathrm{H})$, where $d_{n}=\operatorname{dim}\left(Q_{n}(\mathrm{H})\right)$. Then,

$$
\begin{aligned}
v_{\phi, \psi, Q}^{(N, n)}\left(\times_{\ell=1}^{n} I_{\ell} \times J_{\ell}\right) & :=\left\langle\phi \mid P_{I_{1}}^{(A)} Q_{n} P_{J_{1}}^{(B)} \cdots P_{I_{N}}^{(A)} Q_{n} P_{J_{N}}^{(B)} \psi\right\rangle \\
& =\sum_{k_{1}, \ldots, k_{2 N-1} \leq d_{n}}\left\langle\phi \mid P_{I_{1}}^{(A)} h_{k_{1}}\right\rangle\left\langle h_{k_{1}} \mid P_{J_{1}}^{(B)} h_{k_{2}}\right\rangle \cdots\left\langle h_{k_{2 N-1}} \mid P_{J_{N}}^{(B)} \psi\right\rangle
\end{aligned}
$$

Let us consider the unitary maps $U^{(A)}: \mathrm{H} \rightarrow L^{2}\left(\mathbb{N} \times \mathbb{R}, v^{(A)}\right), U^{(B)}: \mathrm{H} \rightarrow L^{2}(\mathbb{N} \times$ $\left.\mathbb{R}, v^{(B)}\right)$ defined by the spectral representation of the self-adjoint operators $A, B$ as in Theorem B.1. Setting $h_{j}^{(A)}(r):=\left(U^{(A)} h_{j}\right)(r)$ and $h_{j}^{(B)}(s):=\left(U^{(B)} h_{j}\right)(s)$, where $r, s \in \Gamma:=\mathbb{N} \times \mathbb{R}$ the latter equipped with the product measure when the topology on $\mathbb{N}$ is the discrete one, we have,

$$
\begin{gathered}
v_{\phi, \psi, Q}^{(N, n)}\left(\times_{\ell=1}^{n} I_{\ell} \times J_{\ell}\right)=\sum_{k_{1}, \ldots, k_{2 N-1} \leq d_{n}}\left\langle\phi^{(A)} \mid 1_{I_{1}} h_{k_{1}}^{(A)}\right\rangle\left\langle h_{k_{1}}^{(B)} \mid 1_{J_{1}} h_{k_{2}}^{(B)}\right\rangle \cdots\left\langle h_{k_{2 N-1}}^{(B)} \mid 1_{J_{N}} \psi^{(B)}\right\rangle \\
=\int_{(\mathbb{N} \times \mathbb{R})^{2 N}} 1_{\mathbb{N} \times I_{1} \times \cdots \times \mathbb{N} \times J_{N}}\left(r_{1}, \ldots, s_{N}\right) \rho_{\phi, \psi, Q}^{(n)}\left(r_{1}, \ldots, s_{N}\right) \mathrm{d} v^{(A)}\left(r_{1}\right) \cdots \mathrm{d} v^{(B)}\left(s_{N}\right),
\end{gathered}
$$

where we defined $\phi^{(A)}:=U^{(A)} \phi, \psi^{(B)}:=U^{(B)} \psi$ and

$$
\rho_{\phi, \psi, Q}^{(n)}\left(r_{1}, \ldots, s_{N}\right):=\sum_{k_{1}, \ldots, k_{2 N-1} \leq d_{n}} \overline{\phi^{(A)}\left(r_{1}\right)} h_{k_{1}}^{(A)}\left(r_{1}\right) \overline{h_{k_{1}}^{(B)}\left(s_{1}\right)} \cdots \overline{h_{k_{2 N-1}}^{(B)}\left(s_{N}\right)} \psi^{(B)}\left(s_{N}\right) .
$$


Each factor on the right-hand side (like $\overline{\phi^{(A)}\left(r_{1}\right)} h_{k_{1}}^{(A)}\left(r_{1}\right)$ ) is made of a product of a pair of $L^{2}$ functions with the same argument $\left(r_{1}\right)$; therefore, it is an element of $L^{1}$ with respect to the corresponding measure $v^{(A)}$. Therefore, $\rho_{\phi, \psi, Q}^{(n)} \in L^{1}((\mathbb{N} \times$ $\left.\mathbb{R})^{2 N},\left(v^{(A)} \otimes v^{(B)}\right)^{\otimes N}\right)$ because it is a finite sum of $L^{1}$ functions in the product measure. Hence, the set function $\mu_{\phi, \psi, Q}^{(N, n)}: \mathscr{B}\left(\Gamma^{2 N}\right) \rightarrow \mathbb{C}$ defined as

$$
\mu_{\phi, \psi, Q}^{(N, n)}(F):=\int_{F} \rho_{\phi, \psi, Q}^{(n)}\left(r_{1}, \ldots, s_{N}\right) \mathrm{d} v^{(A)}\left(r_{1}\right) \cdots \mathrm{d} \nu^{(B)}\left(s_{N}\right), \quad F \in \mathscr{B}\left(\Gamma^{2 N}\right),
$$

is a $\sigma$-additive complex measure with total variation $\left|\mu_{\phi, \psi, Q}^{(N, n)}\right|\left(\Gamma^{2 N}\right) \leq\left\|\rho_{\phi, \psi, Q}^{(n)}\right\|_{L^{1}}$. Let $\Pi: \Gamma^{2 N} \rightarrow \mathbb{R}^{2 N}$ be the surjective map defined by

$$
\Pi\left(m_{1}, \lambda_{1}, \ldots, m_{N}, \lambda_{N}, l_{1}, \mu_{1}, \ldots, l_{N}, \mu_{N}\right):=\left(\lambda_{1}, \ldots, \lambda_{N}, \mu_{1}, \ldots, \mu_{N}\right),
$$

where $m_{1}, \ldots, m_{N}, l_{1}, \ldots, l_{N} \in \mathbb{N}$, while $\lambda_{1}, \ldots, \lambda_{N}, \mu_{1}, \ldots, \mu_{N} \in \mathbb{R}$. Clearly $\Pi$ is measurable because it is continuous with respect to the natural product topologies. We then define the complex measure $v_{\phi, \psi, Q}^{(N, n)}$ on $\mathscr{B}\left(\mathbb{R}^{2 N}\right)$ extending the values $v_{\phi, \psi, Q}^{(N, n)}\left(\times_{\ell=1}^{n} I_{\ell} \times J_{\ell}\right)$ as the image measure (also called pushforward measure) of $\mu_{\phi, \psi, Q}^{(N, n)}$, namely $v_{\phi, \psi, Q}^{(N, n)}=(\Pi)_{*}\left(\mu_{\phi, \psi, Q}^{(N, n)}\right):=\mu_{\phi, \psi, Q}^{(N, n)} \circ \Pi^{-1}$. With the said definition, if $O \subset \mathbb{R}^{2 N} \backslash \sigma(A) \times \cdots \times \sigma(B)$ is open, then $\Pi^{-1}(O)$ is open and stays in the complement to the set $\mathbb{N} \times \sigma(A) \times \cdots \times \mathbb{N} \times \sigma(B)$. This set includes $\operatorname{supp}\left(\left(v^{(A)} \otimes v^{(B)}\right)^{\otimes N}\right)$ according to (c) Theorem B.1. Hence, $\left(v^{(A)} \otimes v^{(B)}\right)^{\otimes N}\left(\Pi^{-1}(O)\right)=0$. Using (70) observing that $\left(v^{(A)} \otimes v^{(B)}\right)^{\otimes N}$ is a positive measure and $\rho_{\phi, \psi, Q}^{(n)}$ is $L^{1}$ with respect to that measure, we conclude that $\left|\mu_{\phi, \psi, Q}^{(N, n)}\right|\left(\Pi^{-1}(O)\right)=0$ which implies $\left|v_{\phi, \psi, Q}^{(N, n)}\right|(O)=0$. In other words, (22) is true.

(b) Let us define

$$
I_{\phi, \psi}^{(N)}(f):=\int_{\mathbb{R}}\left\langle\phi \mid\left(\mathrm{e}^{i \frac{t}{N} a A} \mathrm{e}^{i \frac{t}{N} b B}\right)^{N} \psi\right\rangle \mathrm{d} v_{f}(t)
$$

where $v_{f}=F^{-1}(f)$ referring to (16). An elementary inductive argument establishes that

$$
S_{1} Q_{n} S_{2} Q_{n} \cdots Q_{n} S_{N} \rightarrow S_{1} S_{2} \cdots S_{N} \quad \text { strongly as } n \rightarrow+\infty
$$

if (1) the orthogonal projectors $Q_{n}$ satisfy $Q_{n} \rightarrow I$ in the strong sense and (2) $S_{1}, \ldots, S_{N} \in \mathfrak{B}(\mathrm{H})$ satisfy $\left\|S_{j}\right\| \leq c<+\infty$ for $j=1, \ldots, N$. Taking advantage of this fact (noticing that $\mathrm{e}^{i s C}$ is unitary if $s \in \mathbb{R}$ and $C$ is self-adjoint, so that $\left\|\mathrm{e}^{i s C}\right\|=1$ ), Lebesgue's dominated convergence theorem and the Fubini theorem allow us to write

$$
I_{\phi, \psi}^{(N)}(f)=\int_{\mathbb{R}}\left\langle\phi \mid \mathrm{e}^{i \frac{t}{N} a A} \mathrm{e}^{i \frac{t}{N} b B} \cdots \mathrm{e}^{i \frac{t}{N} a A} \mathrm{e}^{i \frac{t}{N} b B} \psi\right\rangle \mathrm{d} v_{f}(t)
$$




$$
\begin{aligned}
& =\int_{\mathbb{R}}\left\langle\phi \mid \lim _{n \rightarrow \infty} \mathrm{e}^{i \frac{t}{N} a A} Q_{n} e^{i \frac{t}{N} b B} \ldots \mathrm{e}^{i \frac{t}{N} a A} Q_{n} e^{i \frac{t}{N} b B} \psi\right\rangle \mathrm{d} \nu_{f}(t) \\
& =\lim _{n \rightarrow \infty} \int_{\mathbb{R}}\left\langle\phi \mid \mathrm{e}^{i \frac{t}{N} a A} Q_{n} e^{i \frac{t}{N} b B} \ldots \mathrm{e}^{i \frac{t}{N} a A} Q_{n} e^{i \frac{t}{N} b B} \psi\right\rangle \mathrm{d} \nu_{f}(t) \\
& =\lim _{n \rightarrow \infty} \int_{\mathbb{R}} \int_{\mathbb{R}^{2 N}} \mathrm{e}^{i \frac{t}{N} \sum_{l=1}^{N}\left(a \lambda_{l}+b \mu_{l}\right)} \mathrm{d} \nu_{\phi, \psi, Q}^{N, n}\left(\lambda_{1}, \mu_{1}, \ldots, \lambda_{N}, \mu_{N}\right) \mathrm{d} \nu_{f}(t) \\
& =\lim _{n \rightarrow \infty} \int_{\mathbb{R}^{2 N}} f\left(\frac{1}{N} \sum_{l=1}^{N}\left(a \lambda_{l}+b \mu_{l}\right)\right) \mathrm{d} v_{\phi, \psi, Q}^{(N, n)}\left(\lambda_{1}, \mu_{1}, \ldots, \lambda_{N}, \mu_{N}\right)
\end{aligned}
$$

Due to (71), the limit does not depend on the chosen sequence $Q$ as declared in the hypothesis.

\section{Appendix B: Spectral representation theorem}

We state and prove here a version of the spectral representation theorem for self-adjoint operators we exploited in the proof of Lemma 2.2. This is nothing but a refinement of part of some results which can be found in [25,33].

Theorem B.1 Let $T: D(T) \rightarrow H$ a self-adjoint operator in the separable Hilbert space $H$. The following facts are true.

(a) There is a measure space $(\Gamma, \Sigma, v)$, where

(i) $\Gamma:=\mathbb{N} \times \mathbb{R}$

(ii) $\Sigma:=\mathscr{B}(\Gamma)$ referred to the product topology of $\mathbb{N} \times \mathbb{R}$, equipping $\mathbb{N}$ with the discrete topology,

(iii) $v$ is a positive $\sigma$-additive Borel measure over $\Gamma$ which can be chosen to be finite.

(b) There is a unitary map $U: H \rightarrow L^{2}(\Gamma, \Sigma, v)$, such that the $P V M P^{(T)}$ of $T$ satisfies

$$
\left(U P_{E}^{(T)} \psi\right)(n, x)=1_{E}(x)(U \psi)(n, x) \text { for every } E \in \mathscr{B}(\mathbb{R}),(n, x) \in \Gamma .
$$

(c) It holds $\operatorname{supp}(v) \subset \mathbb{N} \times \sigma(T)$.

Proof (a) and (b). If $\mathrm{H}=\{0\}$ everything is trivial, so that we assume this is not the case. Take $\psi_{0} \in \mathrm{H} \backslash\{0\}$ and define the subspace $\mathrm{H}_{0}:=\left\{f(T) \psi_{0} \mid f \in L^{2}\left(\mathbb{R}, v_{\psi_{0}}\right)\right\}$ where henceforth $v_{\psi}(E):=\left\langle\psi \mid P_{E}^{(T)} \psi\right\rangle=\left\|P_{E}^{(T)} \psi\right\|^{2}$. The linear map

$$
V_{0}: L^{2}\left(\mathbb{R}, v_{\psi_{0}}\right) \ni f \mapsto f(T) \psi_{0} \in \mathrm{H}_{0}
$$

is evidently isometric because $\left\|f(T) \psi_{0}\right\|^{2}=\|f\|_{L^{2}\left(\mathbb{R}, v_{\psi_{0}}\right)}^{2}$ from the elementary spectral theory [25], and surjective by construction. Furthermore, $\mathrm{H}_{0}$ is closed: If 
$\mathrm{H} \ni \psi=\lim _{n \rightarrow+\infty} f_{n}(T) \psi_{0}$, then $\left\{f_{n}\right\}_{n \in \mathbb{N}}$ is Cauchy in $L^{2}\left(\mathbb{R}, v_{\psi_{0}}\right)$ and its limit $f \in L^{2}\left(\mathbb{R}, v_{\psi_{0}}\right)$ satisfies $f(T) \psi_{0}=\psi$. Now take $\psi_{1} \in \mathrm{H}_{0}^{\perp}$ with $\psi_{1} \neq 0$, if there is such vector, and construct an analogous isometric map

$$
V_{1}: L^{2}\left(\mathbb{R}, v_{\psi_{1}}\right) \ni f \mapsto f(T) \psi_{1} \in \mathrm{H}_{1}
$$

observing that $\mathrm{H}_{1} \perp \mathrm{H}_{0}$, because $\left\langle f(T) \psi_{1} \mid g(T) \psi_{0}\right\rangle=\int_{\mathbb{R}} g(\lambda) \mathrm{d} \nu_{f(T) \psi_{1}, \psi_{0}}(\lambda)$, but again from the elementary spectral theory,

$$
\begin{aligned}
v_{f(T) \psi_{1}, \psi_{0}}(E) & :=\left\langle f(T) \psi_{1} \mid P_{E} \psi_{0}\right\rangle=\left\langle f(T) \psi_{1} \mid 1_{E}(T) \psi_{0}\right\rangle \\
& =\left\langle\psi_{1} \mid\left(\bar{f} \cdot 1_{E}\right)(T) \psi_{0}\right\rangle=0
\end{aligned}
$$

We can iterate the procedure finding a class of mutually orthogonal closed subspaces $\left\{\mathrm{H}_{j}\right\}_{j \in \mathbb{N}}$ and a corresponding sequence of isometric surjective maps $V_{j}$ : $L^{2}\left(\mathbb{R}, v_{\psi_{j}}\right) \rightarrow \mathrm{H}_{j}$, where possibly $\mathrm{H}_{j}=\{0\}$ and $v_{\psi_{j}}=0$ from some $j_{0}$ on. We assume that $\psi_{j} \neq 0$ for all $j \in \mathbb{N}$ since the other case is trivial. Since $\mathrm{H}$ is separable, with a straightforward application of Zorn's lemma, we have that the countable Hilbert orthogonal decomposition holds $\mathrm{H}=\oplus_{j=0}^{+\infty} \mathrm{H}_{j}$. As a consequence, $\mathrm{H}$ turns out to be isomorphic to $\bigoplus_{j=0}^{+\infty} L^{2}\left(\mathbb{R}, \mathrm{d} \nu_{\psi_{j}}\right)$ and the isomorphism is nothing but $U^{\prime}:=\oplus_{j=0}^{+\infty} V_{j}^{-1}: \oplus_{j=0}^{+\infty} \mathrm{H}_{j} \rightarrow \oplus_{j=0}^{+\infty} L^{2}\left(\mathbb{R}, \mathrm{d} \nu_{\psi_{j}}\right)$. Notice that taking advantage of the spectral identity $P_{E}^{(T)}=1_{E}(T)$, we have

$$
U^{\prime} P_{E}^{(T)} \psi=\oplus_{j=0}^{+\infty} 1_{E} \cdot\left(V_{j}^{-1} Q_{j} \psi\right)
$$

where $Q_{j}: \mathrm{H} \rightarrow \mathrm{H}$ is the orthogonal projector onto $\mathrm{H}_{j}$. At this juncture, we are free to change the normalization of the vectors $\psi_{j}$ without affecting the found subspaces $\mathrm{H}_{j}$, requiring that $\left\|\psi_{j}\right\|^{2}=2^{-j}$. With the given definitions, if $\Gamma:=\mathbb{N} \times \mathbb{R}, \Sigma:=$ $\mathscr{B}(\mathbb{N} \times \mathbb{R})$, and $\mathbb{R}_{n}:=\{n\} \times \mathbb{R}$, we construct the Borel measure over $\Gamma$

$$
\nu\left(\cup_{n} E_{n}\right):=\sum_{n=0}^{+\infty} v_{\psi_{n}}\left(E_{n}\right), \quad \text { for } E_{n} \in \mathscr{B}\left(\mathbb{R}_{n}\right) \equiv \mathscr{B}(\mathbb{R})
$$

Observe that every $E \in \mathscr{B}(\mathbb{N} \times \mathbb{R})$ uniquely decomposes $E=\cup_{n} E_{n}$ with $E_{n} \in$ $\mathscr{B}\left(\mathbb{R}_{n}\right)$, since $\mathbb{R}_{n} \cap \mathbb{R}_{m}=\varnothing$ for $n \neq m$. Noticing that all measures $v_{\psi_{n}}$ are positive and $\sigma$-additive, it is not difficult to establish that $v$ is such. In particular, $v$ is finite if we choose $\left\|\psi_{j}\right\|^{2}=2^{-j}$, because $v(\Gamma)=\sum_{n=0}^{+\infty} 2^{-n}=2<+\infty$. Finally, it is evident that $f \in L^{2}(\Gamma, \Sigma, v)$ if and only if $f(n, \cdot) \in L^{2}\left(\mathbb{R}, \nu_{\psi_{n}}\right)$ for all $n \in \mathbb{N}$ and $\sum_{n=0}^{+\infty}\|f(n, \cdot)\|_{L^{2}\left(\mathbb{R}, v_{\psi_{n}}\right)}^{2}<+\infty$. In particular,

$$
\|f\|_{L^{2}(\Gamma, \Sigma, v)}^{2}=\sum_{n=0}^{+\infty}\|f(n, \cdot)\|_{L^{2}\left(\mathbb{R}, v_{\psi_{n}}\right)}^{2},
$$


so that there is a unitary map $U^{\prime \prime}: \oplus_{j=0}^{+\infty} L^{2}\left(\mathbb{R}, \mathrm{d} v_{\psi_{j}}\right) \rightarrow L^{2}(\Gamma, \Sigma, v)$, obviously constructed. The wanted Hilbert-space isomorphism $U$ is therefore

$$
U:=U^{\prime \prime} U^{\prime}: \mathrm{H} \rightarrow L^{2}(\Gamma, \Sigma, \nu) .
$$

With this definition, (73) is a direct rephrasing of (74).

(c) It easily arises from the standard result $\operatorname{supp}\left(P^{(T)}\right)=\sigma(T)$, using $\operatorname{supp}\left(v_{\psi}\right) \subset$ $\operatorname{supp}\left(P^{(T)}\right)$ and the disjoint decomposition of $\Gamma$ into open-closed subsets $\mathbb{R}_{n}$.

\section{Appendix C: Projective limit of complex measures}

We state here a particular case of a general result extensively discussed in [2,41], concerning an extension to complex measures of Kolmogorov theorem for the existence of a so-called consistent family of probability measures (see, e.g., [30,43-45]). Let $\mathbb{N}$ be the index set and let us denote with $\mathscr{F}_{\mathbb{N}}$ the collection of subsets $J \subset \mathbb{N}$ with a finite number of elements and with $|J|$ the cardinality of $J \in \mathscr{F}_{\mathbb{N}}$.

$\mathscr{F}_{\mathbb{N}}$ inherits naturally the structure of a directed set, where the partial order relation $\leq$ is defined as $J \leq K$ iff $J \subset K, J, K \in \mathscr{F}_{\mathbb{N}}$. Let us associate with any $J \in \mathscr{F}_{\mathbb{N}}$ a measure space $\mathbb{R}^{J}$, naturally isomorphic to $\mathbb{R}^{|J|}$ and let $\Sigma_{J}$ denote its Borel $\sigma$-algebra. For any pair $J, K \in \mathscr{F}_{\mathbb{N}}$ with $J \leq K$ let $\pi_{J}^{K}: \mathbb{R}^{K} \rightarrow \mathbb{R}^{J}$ be the projection map, which is continuous hence Borel measurable.

A collection $\left\{\mu_{J}\right\}_{J \in \mathscr{F}_{\mathbb{N}}}$ of complex measures $\mu_{J}: \Sigma_{J} \rightarrow \mathbb{C}$ is said to be consistent or projective if the following compatibility condition is fulfilled for any $J, K \in \mathscr{F}_{\mathbb{N}}$, $J \leq K$ :

$$
\mu_{J}=\left(\pi_{J}^{K}\right)_{*} \mu_{K}
$$

where $\left(\pi_{J}^{K}\right)_{*} \mu_{K}$ denotes the image (pushforward) measure of $\mu_{K}$ under the action of the map $\pi_{J}^{K}$, namely $\left(\pi_{J}^{K}\right)_{*} \mu_{K}(E):=\mu_{K}\left(\left(\pi_{J}^{K}\right)^{-1}(E)\right)$, for all $E \in \Sigma_{J}$.

Let $\mathbb{R}^{\mathbb{N}}$ be the space of all real-valued sequences and for any $J \in \mathscr{F}_{\mathbb{N}}$ let $\pi_{J}$ : $\mathbb{R}^{\mathbb{N}} \rightarrow \mathbb{R}^{J}$ indicate the projection map. It is simple to check that for any $J, K \in \mathscr{F}_{\mathbb{N}}$, with $J \leq K$, the following composition property holds:

$$
\pi_{J}=\pi_{J}^{K} \circ \pi_{K}
$$

We shall consider the $\sigma$-algebra $\Sigma$ on $\mathbb{R}^{\mathbb{N}}$ generated by the cylinder sets, i.e., the subsets of the form $\left(\pi_{J}\right)^{-1}(E)$ for some $J \in \mathscr{F}_{\mathbb{N}}$ and $E \in \Sigma_{J}$. It is simple to see that, for every complex measure $\mu: \Sigma \rightarrow \mathbb{C}$, it is possible to define a collections $\left\{\mu_{J}\right\}_{J \in \mathscr{F}_{\mathbb{N}}}$ of complex measures $\mu_{J}: \Sigma_{J} \rightarrow \mathbb{C}$ as the pushforward of $\mu$ under the action of the projections $\pi_{j}$, i.e.,

$$
\mu_{J}:=\left(\pi_{J}\right)_{*} \mu
$$

Further, by the composition property (76), the resulting collection of measures $\left\{\mu_{J}\right\}_{J \in \mathscr{F}_{\mathbb{N}}}$ is consistent, i.e., satisfies condition (75) which is indeed a necessary condition for the existence of a measure $\mu: \Sigma \rightarrow \mathbb{C}$ generating the family $\left\{\mu_{J}\right\}_{J \in \mathscr{F}_{\mathbb{N}}}$ 
through (77). Remarkably, in the case where the measures $\left\{\mu_{J}\right\}_{J \in \mathscr{F}_{\mathbb{N}}}$ are probability measures, according to Kolmogorov existence theorem [30,43-45] the consistency condition (75) is also sufficient for the existence of a probability measure $\mu: \Sigma \rightarrow \mathbb{R}$ such that (77) holds. This result is a cornerstone of probability theory, actually providing the existence of stochastic processes. In the case where the measures $\left\{\mu_{J}\right\}_{J \in \mathscr{F}_{\mathbb{N}}}$ belonging to the projective family are complex (or signed) measures, the classical Kolmogorov theorem can no longer be directly applied. Indeed, in this case, additional conditions have to be required and we refer to [41] for details. In particular, a fundamental condition is the following upper bound on the total variation of the measures belonging to the family $\left\{\mu_{J}\right\}_{J \in \mathscr{F}_{\mathbb{N}}}$ :

$$
\sup _{J}\left\|\mu_{J}\right\|<\infty, \quad\left\|\mu_{J}\right\|:=\left|\mu_{J}\right|\left(\mathbb{R}^{J}\right) .
$$

Indeed, the necessity of condition (78) can be simply proved by observing that whenever $h: X \rightarrow Y$ is a measurable map between two measurable spaces $\left(X, \Sigma_{X}\right)$ and $\left(Y, \Sigma_{Y}\right)$ and for any complex measure $\mu$ on $\left(X, \Sigma_{X}\right)$, the total variation of the pushforward measure $(h)_{*} \mu$ on $\left(Y, \Sigma_{Y}\right)$ satisfies the following inequality ${ }^{8}$

$$
\left\|(h)_{*} \mu\right\| \leq\|\mu\| .
$$

Hence, by Eq. (77), for any $J \in \mathscr{F}_{\mathbb{N}}$ we obtain

$$
\left\|\mu_{J}\right\|=\left\|\left(\pi_{J}\right)_{*} \mu\right\| \leq\|\mu\|,
$$

which yields (78).

\section{References}

1. Albeverio, S., Mazzucchi, S.: A unified approach to infinite-dimensional integration. Rev. Math. Phys. 28(2), 1650005-43 (2016)

2. Albeverio, S., Guatteri, G., Mazzucchi, S.: Phase space Feynman path integrals. J. Math. Phys. 43(6), 2847-2857 (2002)

3. Araki, H.: Expansional in Banach algebras Annales scientifiques de l'E.N.S. 4 e serie, tome 6(1), 67-84 (1973)

4. Beltrametti, E.G., Cassinelli, G.: The logic of quantum mechanics. In: Encyclopedia of Mathematics and Its Applications, vol. 15. Addison-Wesley, Reading, MA (1981)

5. Billingsley, P.: Probability and Measure. Wiley Series in Probability and Statistics. Wiley, Hoboken (2012)

6. Cassinelli, G., Lahti, P.: Quantum mechanics: why complex Hilbert space? Philos. Trans. R. Soc. A Math. Phys. Eng. Sci. 375, 02 (2017)

7. Cohn, D.L.: Measure Theory. Birkhäuser, Boston (1980)

$\overline{8 \text { For any partition }\left\{E_{j}\right\}_{j} \subset \Sigma_{Y}}$ of $Y$, we have that the collection of sets $\left\{h^{-1}\left(E_{j}\right)\right\}_{j} \subset \Sigma_{X}$ is a partition of $X$. Hence

$$
\sum_{j}\left|(h)_{*} \mu\left(E_{j}\right)\right|=\sum_{j}\left|\mu\left(h^{-1}\left(E_{j}\right)\right)\right| \leq\|\mu\| .
$$


8. Dao-Xing, X., Brody, E.J.: Measure and Integration Theory on Infinite Dimensional Spaces. Academic Press, New York (1972)

9. Dvurecenskij, A.: Gleason's Theorem and Its Applications. Kluwer Academic Publishers, Dordrecht (1992)

10. Emch, G.G.: Algebraic Methods in Statistical Mechanics and Quantum Field Theory. Wiley, New York (1972)

11. Gill, T.L., Zachary, W.W.: Functional Analysis and the Feynman Operator Calculus. Springer, Berlin (2016)

12. Gleason, A.M.: Measures on the closed subspaces of a Hilbert space. J. Math. Mech. 6, 885-893 (1957)

13. Gudder, S.: Some unsolved problems in quantum logic. In: Marlow, A.R. (ed.) Mathematical Foundations of Quantum Theory. Academic Press (1978)

14. Guerra Bobo, I.: On quantum conditional probability. Theoria 28, 115-137 (2013)

15. Hamhalter, J.: Quantum Measure Theory. Springer, Berlin (2003)

16. Johnson, G.W., Lapidus, M.L.: The Feynman Integral and Feynman's Operational Calculus. Clarendon Press, Oxford (2000)

17. Johnson, G.W., Lapidus, M.L., Nielsen, L.: Feynman's Operational calculus and Beyond. Noncommutativity and Time-Ordering. Oxford University Press, Oxford (2015)

18. Kumano-go, N.: Phase space Feynman path integrals with smooth functional derivatives by time slicing approximation. Bull. Sci. Math. 135(8), 936-987 (2011)

19. Landsman, K.: Foundations of Quantum Theory. Springer, Berlin (2017)

20. Lapidus, M.L.: The differential equation for the Feynman-Kac formula with a Lebesgue-Stieltjes measure. Lett. Math. Phys. 11, 1-13 (1986). (Special issue dedicated to the memory of Mark Kac)

21. Lapidus, M.L.: The Feynman-Kac formula with a Lebesgue-Stieltjes measure and Feynman's operational calculus. Stud. Appl. Math. 76, 93-132 (1987)

22. Lapidus, M.L.: The Feynman-Kac formula with a Lebesgue-Stieltjes measure: an integral equation in the general case. Integral Equ. Oper. Theory 12, 162-210 (1989)

23. Mackey, G.: The Mathematical Foundations of Quantum Mechanics. Benjamin, New York (1963)

24. Maslov, V.P.: Operational Methods. Mir Publishers, Moscow (1976). English translation

25. Moretti, V.: Spectral Theory and Quantum Mechanics, 2nd Revised and Enlarged Edition. Springer, Berlin (2017)

26. Moretti, V., Oppio, M.: The correct formulation of Gleason's theorem in quaternionic Hilbert spaces. Ann. Henri Poincaré 19, 3321-3355 (2018)

27. Nazaikinskii, V.E., Shatalov, V.E., Sternin, B.Y.: Methods of Noncommutative Analysis: Theory and Applications. Walter de Gruyter, Berlin (1996)

28. Nelson, E.: Operants: a functional calculus for non-commuting operators. In: Browder, F.E. (ed.) Functional Analysis and Related Fields, pp. 172-187. Springer, Berlin (1970)

29. Nielsen, L.: Combining continuous and discrete phenomena for Feynman's operational calculus in the presence of a $(C C 0)$ semigroup and Feynman-Kac formulas with Lebesgue-Stieltjes measures. Integr. Equ. Oper. Theory 90, 12 (2018). https://doi.org/10.1007/s00020-018-2428-8

30. Prokhorov, YuV: Convergence for random processes and limit theorems in probability theory. Theory Prob. Appl. 1, 177-238 (1956). (transl.)

31. Redéi, M.: Quantum Logic in Algebraic Approach. Kluwer Academic Pub, Dordrecht (1998)

32. Reed, M., Simon, B.: Methods of Modern Mathematical Physics, vol II Fourier Analysis, SelfAdjointness. Academic Press, Cambridge (1975)

33. Reed, M., Simon, B.: Methods of Modern Mathematical Physics, Functional Analysis Revised and Enlarged Edition, vol. I. Academic Press, Cambridge (1980)

34. Reznick, B.: Some new canonical forms for polynomials. Pac. J. Math. 266, 185-220 (2013)

35. Rudin, W.: Real and Complex Analysis, 2nd print. McGraw-Hill Book Company, New York (1987)

36. Rudin, W.: Functional Analysis, 2nd edn. McGraw-Hill Book Company, New York (1993)

37. Schmüdgen, K.: Unbounded Self-Adjoint Operators on Hilbert Space, vol. 265. Springer, Berlin (2012)

38. Simon, B.: Functional Integration and Quantum Physics, 2nd edn. AMS Chelsea Publishing, Providence (2005)

39. Streater, R.F.: Lost Causes in and Beyond Physics. Springer, Berlin (2007)

40. Strocchi, F.: An Introduction to the Mathematical Structure of Quantum Mechanics: A Short Course for Mathematicians. World Scientific, Singapore (2005)

41. Thomas, E.: Projective limits of complex measures and martingale convergence. Probab. Theory Relat. Fields 119(4), 579-588 (2001) 
42. Varadarajan, V.S.: Geometry of Quantum Theory, 2nd edn. Springer, Berlin (2007)

43. von Neumann, J.: Mathematische Grundlagen der Quantenmechanik. Springer, Berlin (1932)

44. Yamasaki, Y.: Measures on Infinite Dimensional Spaces. World Scientific, Singapore (1985)

45. Yeh, J.: Stochastic Processes and the Wiener Integral. Marcel Dekker Inc, New York (1973)

Publisher's Note Springer Nature remains neutral with regard to jurisdictional claims in published maps and institutional affiliations. 